\title{
Syntax through the looking glass: A review on two-word linguistic processing across behavioral, neuroimaging and neurostimulation studies
}

\author{
Matteo Maran ${ }^{1,2}$, Angela D. Friederici ${ }^{1}$, and Emiliano Zaccarella ${ }^{1}$ \\ ${ }^{1}$ Max Planck Institute for Human Cognitive and Brain Sciences, Leipzig, Germany \\ ${ }^{2}$ IMPRS NeuroCom, Leipzig, Germany
}

\begin{abstract}
In recent years a growing number of studies on syntactic processing has employed basic two-word constructions (e.g., "the tree") to characterize the fundamental aspects of linguistic composition. This large body of evidence allows, for the first time, to closely examine which cognitive processes and neural substrates support the combination of two syntactic units into a more complex one, mirroring the nature of combinatory operations described in theoretical linguistics. The present review comprehensively examines behavioral, neuroimaging and neurostimulation studies investigating basic syntactic composition, covering more than 40 years of psycho- and neuro-linguistic research. Across several paradigms, four key features of syntactic composition have emerged: (1) the rule-based and (2) automatic nature of the combinatorial process, (3) a central role of Broca's area and the posterior temporal lobe in representing and combining syntactic features, and (4) the reliance on efficient bottom-up integration rather than top-down prediction.
\end{abstract}

\section{Introduction}

Scrolling through a dictionary, we easily notice that the list of entries corresponds to a large set of different meanings (tree, eat, green, etc.), but to a few grammatical categories (e.g., noun, verb, adjective, etc.). It is suggested that what enables us to produce and comprehend a virtually infinite number of linguistic expressions is based upon the capacity to combine the few available grammatical categories into abstract hierarchical structures, according to grammar (Berwick et al., 2013, Everaert et al., 2015). As an example, the adjective

\section{Corresponding author:}

Name: Matteo Maran

Email address: maran@cbs.mpg.de

Correspondence address: Max Planck Institute for Human Cognitive and Brain Sciences, Stephanstraße 1a, 04103, Leipzig, Germany big and the noun tree can be combined to form the noun phrase big tree, in accordance with the syntactic rules of the English language. Conceptual-semantic information fills the abstract hierarchical structure with meanings from the lexicon, integrating the semantic information of individual words into more complex ones-e.g., the entity size denoted by big and the entity itself denoted by tree.

Characterizing the neural basis of language composition is a central focus of the most recent research programs in neurolinguistics (Fedorenko et al., 2016, Friederici et al., 2017; Hagoort, 2015, Martin \& Baggio, 2020, Pylkkänen, 2019. Zaccarella \& Friederici, 2017). Functional studies have provided evidence for a left-lateralised fronto-temporal network involved in language comprehension, composed of the inferior frontal gyrus (IFG), the ventromedial portion of the prefrontal cortex (vmPFC), the anterior, middle, and posterior sections of the temporal lobe (ATL, MTL and PTL), and the angular gyrus (AG; Chen et al., 2021; Fedorenko et al., 2020; Fedorenko et al., 2016; Goucha and Friederici, 2015, Hagoort, 2015, Hagoort and Indefrey, 2014, Matchin et al., 2017; Matchin and Hickok, 2020, Pallier et al., 2011; Schell et al., 2017, Zaccarella, Meyer, et al., 2017, Zaccarella, Schell, et al., 2017). In recent years, a few functional studies have begun to employ simple two-word combinations to more closely focus on the fundamental question of how linguistic features are combined at the most basic level within the language network (Bemis \& Pylkkänen, 2011; Zaccarella \& Friederici, 2015a). There is general agreement that linguistic processes driven by conceptual-semantic information particularly involve the ATL, the vmPFC, and the AG (Graessner, Zaccarella, Friederici, et al., 2021; Graessner, Zaccarella, \& Hartwigsen, 2021), as also discussed in a recent comprehensive review (Pylkkänen, 2020) specifically addressing the so-called "red boat" paradigm (Bemis \& Pylkkänen, 2011).

The way syntactic information controls linguistic processing during basic combination has received increasing attention in recent years, through a growing number of behavioral and neuroimaging studies. This growing body of evidence, which is set out to highlight the central features of the syntactic combinatorial system, and which can provide fundamental guidance to the interpretation of data from longer 
structures, still awaits systematic examination. Moreover, the only existing review on simple syntactic combination has been published more that twenty-five years ago (Münte \& Heinze, 1994), and cannot include any more recent work. In order to provide a comprehensive overview of what we know about simple syntactic combinations, the purpose of the present paper is to systematically examine all available evidence from studies that use two-word paradigms to test how syntactic information is used to form more complex linguistic representations. The choice to look at the very basic two-word level is motivated by a number of reasons:

- Detailed level of observation: The two-word level allows to isolate the neural correlates of a single application of a compositional operation (Bemis \& Pylkkänen, 2011; Zaccarella \& Friederici, 2015a), which are otherwise observed only indirectly when employing more complex sentence materials. Crucially, two words are indeed sufficient to appreciate the syntactic compositional engine. For example, a determiner phrase (e.g., "The cat") already reflects the application of a syntactic rule (Determiner, Noun $\rightarrow$ Determiner Phrase).

- Fitting the level of observation to linguistic theory: At the syntactic level, the binding mechanism which combines words into phrases (called Merge in theoretical linguistics, Chomsky, 1995) is a binary one (i.e., operates on two elements). Therefore, from a theoretical point of view, the two-word level closely matches the properties of the basic compositional mechanism. Furthermore, as complex structures are the result of the recursive application of Merge (Chomsky, 1995), the two-word level might be sufficient to capture the key aspects of syntactic composition.

- Cross-linguistic and cross-population comparison: The use of two-word constructions greatly reduces the need for additional processes required for the analysis of long structures (e.g., working memory), which otherwise must be subtracted by careful experimental manipulations (Iwabuchi et al., 2019; Makuuchi et al., 2009). Beyond the two-word level, separating pure compositional operations from these processes without introducing additional confounding might be possible in some languages (e.g., languages with free word order) but not in others. This could hinder crosslanguage comparisons and, ultimately, the search for generalizable combinatorial effects. In addition, the use of paradigms with limited extra-linguistic demands makes it possible to study composition in populations whose cognitive reserve might differ from healthy adults. For example, language composition can be studied and compared in young and old populations (Poulisse et al., 2020; Poulisse et al., 2019), distinguishing its developmental trajectory from the acquisition and decline of general domain processes. Similarly, brain damage can be linked to specific linguistic deficits (Graessner, Zaccarella, Friederici, et al., 2021).

- Cross-study comparison: Despite the minimal structures involved, a wide range of detailed linguistic hypotheses can be tested by employing only two words, with the results from different experiments becoming easily comparable. An example is offered by the research program described by Pylkkänen, 2020, where the use of two-word stimuli was kept constant and the dimension of composition was manipulated in a step-wise fashion across studies. This structured series of studies provided great insights into the conceptual compositional processes occurring in the ATL, portraying precisely which types of representation are combined by this region. A similar approach can be adopted to study syntactic composition.

- Isolating top-down and bottom-up processes: The twoword level allows to isolate the relative contribution of top-down and bottom-up processes in language comprehension. In particular, it is possible to employ experimental condition which restrict prediction to the first word and integration to the second one, manipulating selectively one of the two key processes (Maran et al., 2021; Matar et al., 2021). This line of research could provide crucial insights into the neural basis of parsing (Abney \& Johnson, 1991; Resnik, 1992), which at present is tested by comparing topdown, left-corner and bottom-up models of language comprehension in the context of narratives or longer stimuli (Bhattasali et al., 2019, Brennan \& Pylkkänen, 2017; Hale et al., 2018; Nelson et al., 2017).

- Filling a gap in the literature: Systematic reviews on the neuro-cognitive correlates of basic syntactic combination are almost completely missing (Münte \& Heinze, 1994), and mostly focus on studies in which the two-words were presented in the visual modality.

\section{Syntactic composition at the two-word level}

At the two-word level, three main paradigms have been employed to investigate the representations and cognitive processes underlying basic syntactic composition (Figure 1): (1) Categorical and agreement violations, (2) Phrases versus lists contrasts and (3) Noun versus verb contrasts (as part of phrases and sentences, respectively). These paradigms have employed the following eleven languages: Chinese, English, Dutch, Finnish, French, German, Italian, 
Two-word syntactic processing: overview of the existing literature
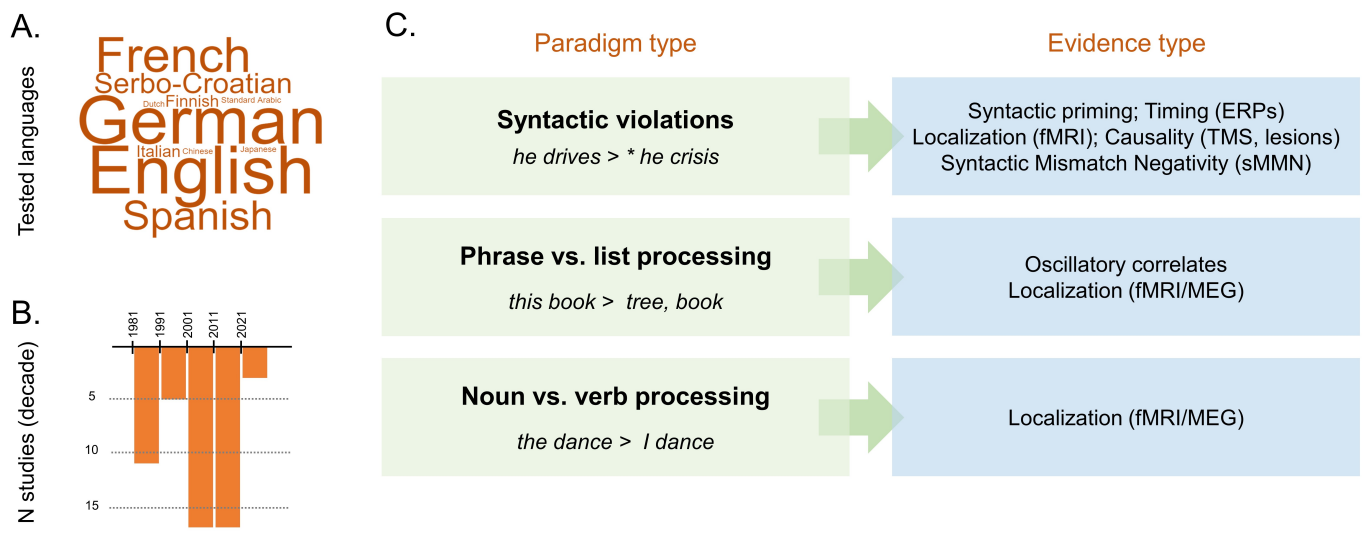

\section{Figure 1}

Overview of the existing literature. (A) Wordcloud representing the relative proportion of the languages tested in the reviewed studies; (B) Temporal evolution of the use of two-word paradigms at the syntactic level; $(C)$ Summary of the paradigms, including examples; (D) Effects of interest highlighted by each of the paradigms.

Japanese, Serbo-Croatian, Spanish, and Standard Arabic. Note that, despite growing interest in the neural basis of signed languages comprehension (Blanco-Elorrieta et al., 2018, Matchin et al., 2022, Moreno et al., 2018, Stroh et al., 2019; Trettenbrein et al., 2020), we are not aware of studies addressing syntactic composition at the "twoword" level in this modality. Therefore, only studies in the visual or auditory modality are included in the present review. Furthermore, we focus on studies investigating comprehension rather than production.

\subsection{Syntactic violations}

Syntactic violations consist of comparing well-formed phrases and sentences with utterances that are ungrammatical, either because of the inability to combine two grammatical categories in a constituent (e.g., "* he boat' 1 ' or due to an agreement error (e.g., "*a boats"). Under the broad definition of syntactic violations, three lines of research can be found:

- Syntactic priming: A first line of behavioral studies investigated whether a minimal syntactic context (e.g., a determiner) can affect the recognition and extraction of linguistic features from an upcoming word (e.g., a noun $\sqrt{2}$. These studies are reviewed in section 2.1.1.

- Syntactic violations (EEG, fMRI, TMS studies): A second line of research employed violations in combination with neuroimaging and/or neurostimulation techniques to characterise the time-course of syntactic analysis and the corresponding functional localization. These studies are reviewed in section 2.1.2.

- Syntactic Mismatch Negativity: Finally, a related line of research investigated the degree of automaticity in syntactic processing, focusing on a specific Event-

${ }^{1}$ Following the convention adopted in theoretical linguistics, we use the asterisk symbol * to denote an ungrammatical construction.

${ }^{2}$ We here use the general term "syntactic priming", as in the first (Goodman et al., 1981) and most recent (Berkovitch \& Dehaene, 2019. Pyatigorskaya et al., 2021) applications of the paradigm. To avoid confusion, we make it explicit here that the effect we describe is different from the structural priming effect (Bock, 1986), sometimes also called "syntactic priming", which refers to the facilitation observed in processing or producing two sentences with the same grammatical structure. 
Related Potential (ERP) component, namely the syntactic mismatch negativity (sMMN). These studies are reviewed in section 2.1.3.

\subsubsection{Syntactic priming}

Rationale. The syntactic priming paradigm consists in the sequential presentation of two words (Figure 2), the first one being the prime (e.g., "the", establishing a syntactic context) and the second one being the target (e.g., "cat"), on which the participants perform a linguistic task (e.g., lexical decision). This paradigm allows to investigate how the certain features introduced by the syntactic context affect the processing of the incoming word, by manipulating the grammatical relationship between the prime and the target. Compared to the use of long sentences, two-word priming allows to keep working memory demands to the minimum. Secondly, confounds at the semantic level are also minimized, especially if function words are used as primes (Goodman et al., 1981). For example, a determiner presented as prime (e.g., "the") does not point to any specific semantic dimension of the target.

A first application of this paradigm is found in the study by Goodman et al. (1981). The authors presented nouns and verbs as target words, preceded by primes which could be determiners (e.g., "the"), possessive adjectives (e.g., "my"), personal pronouns (e.g., "you) or general nouns (e.g., "kids"). Therefore, the relationship between primes and targets could be grammatical (e.g., "you slept", "the flower") or ungrammatical (e.g., "*the slept", “*you flower"). The authors observed faster response times (RTs) in a lexical decision task for targets embedded in grammatical constructions than in ungrammatical ones, when the only contextual effect present in the block was syntactic and when the subjects were instructed regarding the prime-target relationship (Experiment 1). In Experiment 2 the authors tested whether the syntactic priming effect would have still been observed when semantic contextual effects were also presented in the same block. Moreover, subjects' attention was not directed towards the prime-target relationship. Under these circumstances, both syntactic and semantic priming were observed in their respective blocks, but only the latter was observed in the mixed condition. Overall, this study provided initial evidence for the influence of syntactic context in word processing, while leaving open the degree to which this effect was automatic. A limitation of this study was that some of the target words employed might not have been optimal to induce syntactic violations. With one exception, target verbs were presented in the past simple form, to avoid categorical ambiguity (e.g., "slept" instead of "sleep"). However, some of the chosen verbs shared the same form for the past simple and past participle, either as an irregular (e.g., "paid") or regular (e.g., "agreed") form.
Since the past participle can function as an adjectival form, some of the ungrammatical conditions (e.g., "*no agreed") could be in principle still be part of a larger grammatical construction (e.g., "no agreed policy"), in which the past participle serves as a left-side modifier of a noun. This might have diminished the observed syntactic priming effect.

Morphosyntactic features. The results of Goodman et al. (1981) could not be easily explained in terms of spread of activation within the lexicon, contrary to semantic and associative priming effects. Under such an account, a prime (e.g., "he") would lead to the paradoxical diffuse activation of all the members of a category (e.g., verb) in the lexicon (Lukatela, Kostić, Todorović, et al., 1987; Seidenberg et al., 1984). The results of Goodman et al. (1981) inspired a line of studies employing the syntactic priming paradigm, aimed at understanding the computational basis of syntactic contextual effects. Most of these studies were conducted in Serbo-Croatian language, which has a richer inflectional system than English, therefore providing an ideal background to investigate the role of morphology in syntactic priming. A common working hypothesis behind these studies is that syntactic priming might reflect the spread of activation from a prime to a limited number of inflectional morphemes (e.g., $h e$ and $-s$ ), agreeing in syntactic features, rather than to a large number of full lexical entries (Lukatela et al., 1983).

First evidence in support of a crucial role of agreeing morphemes during syntactic priming (e.g., case, person, number) comes from a series of lexical decision tasks showing faster response times (RTs) for case-marked target nouns agreeing with prepositions (Lukatela et al., 1983) and adjectives (Gurjanov, Lukatela, Lukatela, et al., 1985, Experiment 2) presented as primes, compared to ungrammatical prime-target relationships. Similar effects were observed for target verbs agreeing with pronouns presented as primes (Lukatela et al., 1982. Experiments 1 and 2). Importantly, as these studies employed a lexical decision task, they also included pseudowords, both as primes and targets. When presented as targets, pseudo-word 3 included suffixes agreeing or not with the prime (Gurjanov, Lukatela, Moskovljević, et al., 1985; Lukatela et al., 1983; Lukatela et al., 1982), therefore allowing to establish grammatical or ungrammatical relationships. As pseudowords are not elements of the lexicon, any contextual effect observed on these items must depend on grammatical prime-suffix relationship in word processing. In line with a role of prime-suffix relationships, longer RTs were observed for rejecting pseudo-nouns and pseudo-verbs when they syntactically agreed with the prepositions (Lukatela et al., 1983), or pronouns (Lukatela et al., 1982). This pattern of results suggests that the participants were influenced by

\footnotetext{
${ }^{3}$ Pseudo-words are word-like stimuli which respect the orthographical and phonological rules of a language but are not present in its lexicon (e.g., "boal" in English).
} 


\section{Syntactic violations: Masked and unmasked syntactic priming}

A. List of studies

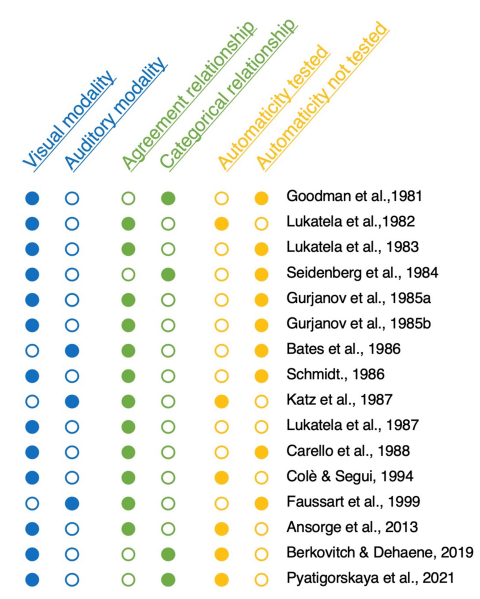

B. Standard experimental paradigm

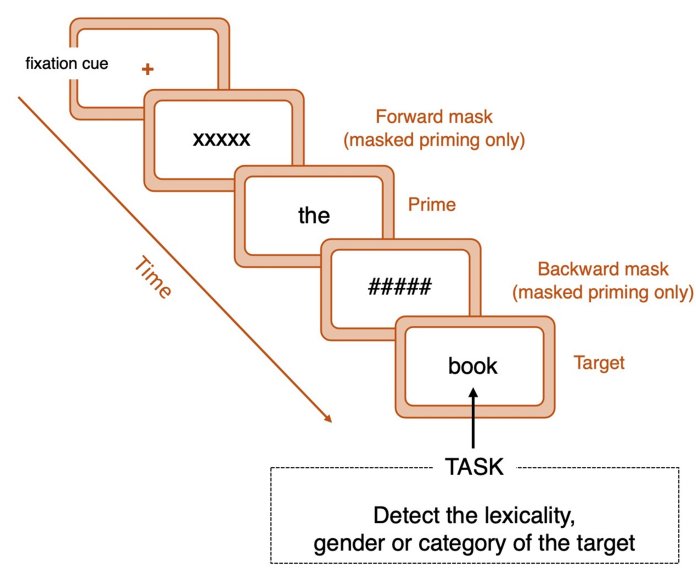

\section{Figure 2}

Syntactic priming studies. (A) Overview of the manipulations; (B) Example of syntactic priming.

the presence or absence of a grammatical relationship with the prime, when evaluating the lexicality of the target.

Preliminary evidence also showed that nouns in the nominative case were not affected by the case agreement with preceding adjectives (Gurjanov, Lukatela, Moskovljević, et al., 1985). A follow-up study tested whether agreement effects could also be observed in the nominative case along the dimension of grammatical gender, rather than case (Gurjanov, Lukatela, Lukatela, et al., 1985). In Experiment 1, Serbo-Croatian adjectives and nouns with typical endings were used (i.e., masculine ending with the null morpheme $\emptyset$, feminine ending with “-a”). Additional pseudo-adjectives and pseudo-nouns with similar endings were also employed, as the participants were performing a lexical decision task on both the prime and the target. The authors observed a syntactic priming effect, with faster RTs on the target when adjectives and nouns were congruent along the gender dimension, regardless of whether the target nouns had typical or atypical endings Overall, this study supports the existence of syntactic priming effects also in the absence of case-marking suffixes, converging on results observed in English at the categorical level (Goodman et al., 1981; Seidenberg et al., 1984). Further evidence for priming of grammatical gender in adjective-noun combinations comes from a study conducted in Italian, with auditory materials
(Bates et al., 1996). In this study, the authors included both targets which were phonologically transparent regarding the gender and opaque along this dimension. Across three different tasks (word repetition, gender monitoring and grammaticality judgment), longer RTs were observed for conditions with gender violations compared to grammatical pairs, independently of phonetic transparency.

All-or-none nature of syntactic priming. The studies of Gurjanov, Lukatela, Lukatela, et al., 1985 and Bates et al., 1996 draw attention to the relevance of the different dimensions along which syntactic agreement takes place (e.g., case, person features, number and grammatical gender). A small number of studies further addressed this aspect, testing whether syntactic priming varies according to the type (e.g., number, gender) and number (single or double) of violations (Faussart et al., 1999; Lukatela, Kostić, Todorović, et al., 1987), or differs between closed-class and open-class elements (Colé \& Segui, 1994). Lukatela, Kostić, Todorović, et al. (1987) employed five types of conditions: one with full agreement between prime and target, three with a single violation (case, gender, or number) and one with a double violation (gender and number). The authors observed that neither the type nor the number of violation(s) affected the magnitude of syntactic priming. This study 
therefore provided initial evidence for an all-or-none nature of syntactic priming, merely reflecting a grammatical or ungrammatical relationship between prime and target. Similar results were observed, across four experiments, in a study conducted in French (Colé \& Segui, 1994), but not in the auditory modality (Faussart et al., 1999). In particular, Faussart et al. (1999) reported larger interference induced by violation of grammatical gender compared to number, both in French (Experiment 1) ${ }^{4}$ and Spanish (Experiment 2). As the authors discussed, these results support the notion that gender but not number is an inherent feature of lexical stems, and that different projections exist between Number and Gender Phrases. A theoretically relevant aspect, which was addressed in only one study (Colé \& Segui, 1994), is whether syntactic priming can be modulated by the type of class (function and content words) of the prime. Given the merely syntactic nature of closed-class elements, it is reasonable to expect larger effects for primes belonging to this category. Indeed, when the prime duration was reduced to approximately $150 \mathrm{~ms}$ (Experiment 3 and 4), closedclass primes elicited a larger syntactic priming effect, even when controlling for frequency occurrence (Experiment 4). However, it remains unclear whether this effect merely reflects length differences between the open and closed-class elements 5 Overall, further research might be needed to shed light on the all-or-none or graded nature of syntactic priming, as methodological differences (e.g., orthogonality of the manipulations, see footnote 4) or different presentation modality (visual or auditory) might have contributed to the diverging results (Colé \& Segui, 1994, Faussart et al., 1999, Lukatela, Kostić, Todorović, et al., 1987).

Pre-lexical versus post-lexical processing. A line of studies tested whether syntactic priming arises at the preor post-lexical level of word processing (Carello et al., 1988. Seidenberg et al., 1984). The distinction between pre-lexical and post-lexical levels refers to whether the prime affected the target word's recognition or a later stage of its processing (e.g., integration). These studies are part of a larger discussion on whether lexical access is an encapsulated process (Forster, 1981) or could be influenced by systems outside of the lexicon (Marslen-Wilson \& Tyler, 1980). To address this research question, researchers built on the properties of two different tasks, i.e. naming and lexical decision, which have been related to manipulations at the pre- and post- lexical stage respectively (West \& Stanovich, 1982). In particular, as lexical decisions occur more slowly than naming, there is sufficient time for the syntactic and message processors to check the congruity of incoming linguistic features with the context, and bias the decision towards a negative outcome in case of a violation (Carello et al., 1988; Seidenberg et al., 1984; West \& Stanovich, 1982). Furthermore, lexical decision can be characterised as a decision making process which can be biased in light of congruity checks (Seidenberg et al., 1984), contrary to naming. Both studies conducted in English (Seidenberg et al., 1984) and Serbo-Croatian (Carello et al., 1988)languages provided evidence for a post-lexical nature of syntactic priming. This aspect converges on the notion that, during language comprehension, the goal is an efficient integration of incoming linguistic information (Seidenberg et al., 1984), which at least at the syntactic level might achieved without predictive processes at the pre-lexical level.

Automaticity. The post-lexical nature of syntactic priming does not necessarily imply that it stems from a non-automatic process. Note that a grammatical relationship between prime and target is not necessary to provide a correct response (i.e., indicating whether the target is a word or not) in the studies described above, but still the participants seemed unable to ignore this information (Faussart et al., 1999; Seidenberg et al., 1984), further pointing towards a strongly automatic process. Studies manipulating the stimulus-onset asynchrony (SOA) and the inter-stimulus interval (ISI) between prime and target (Colé \& Segui, 1994, Katz et al., 1987; Lukatela et al., 1982) provided clear evidence for the automaticity of syntactic priming. The rationale behind these manipulations is that when the target quickly follows the prime there is insufficient time for attentional and strategic processes to influence the response (Lukatela et al., 1982). Interestingly, despite the very short SOAs employed (e.g., 300, 150 and $130 \mathrm{~ms}$ ), syntactic priming has been reliably observed in the literature (Colé \& Segui, 1994, Lukatela et al., 1982).

An additional approach to address the automaticity of syntactic priming is to employ a non-visual modality. As Katz et al. (1987) pointed out, syntactic priming was extensively studied in experiments in which primes and targets were presented visually and with no incrementality (i.e., the entire wordform was presented simultaneously and not letter by letter). In these experiments the subjects might have focused on the suffixes and ignored the stems, therefore inflating the role of morphological markers in word processing. Presenting the stimuli auditorily would therefore allow to control for this potential confound, as the subjects would hear the stem first and the suffix later. Supporting the automatic nature of syntactic priming, shorter RTs in lexical decision task were observed in the auditory modality for target nouns agree-

\footnotetext{
${ }^{4}$ Note that in the study of Colé and Segui (1994) and in the Experiment 1 of Faussart et al. (1999) only masculine targets noun were used, which might be problematic for testing the influence of the gender of the prime on syntactic processing. This issue is addressed in the Experiment 2 of Faussart et al. (1999) with Spanish materials.

${ }^{5}$ Experiment 3: an average of 6 vs 3 letters; Experiment 4: an average of 5.2 vs 4.1 letters.
} 
ing with inflected adjective or pseudo-adjectives presented as primes (Katz et al., 1987). Furthermore, syntactic priming could be observed even with an interval of zero milliseconds between primes and targets (Katz et al., 1987). Syntactic priming effects in the auditory modality were further observed using French and Spanish determiner phrases (Faussart et al., (1999).

More recent studies addressed the automaticity of syntactic priming via the subliminal presentation of the prime word (Ansorge et al., 2013, Berkovitch \& Dehaene, 2019, Pyatigorskaya et al., 2021), using the masking technique to suppress its conscious perception. In a first series of experiments in German, Ansorge et al. (2013) tested whether the gender of a noun could be primed by a masculine or feminine determiner presented subliminally, and if this effect would depend on specific task demands. When participants were engaged in classifying the gender of the target noun (Experiments 1 and 4), shorter RTs were observed for grammatical pairs (i.e., when the gender of the determiner matched the target's one) compared to ungrammatical items. However, when participants were asked to categorize the target noun according to semantic features (Experiment 2), no facilitation for grammatical constructions was observed. The authors suggest that morpho-syntactic processes might be characterized by "conditional automaticity", with task related top-down process at work driving the subliminal effect of the prime. Importantly, as the authors discuss, the primes used were ambiguous regarding the grammatical gender feature, once not only the nominative and singular forms are considered fects of gender-congruence in this study strongly depend on the interpretation of the prime as a nominative singular form. Under an alternative account, it is possible that once the attention of the participant is directed to semantic features of the target, syntactic processing proceeds automatically, considering all the possible interpretations of the two ambiguous primes $^{67}$.

A similar approach to test automaticity, albeit focusing mostly on the categorical level, was employed in a recent study conducted in French by Berkovitch and Dehaene (2019). Participants were asked to indicate the category of the target word (noun or verb), which followed the masked or unmasked presentation of a determiner or a pronoun. Syntactic priming was observed under both masked and unmasked conditions ( $7 \mathrm{~ms}$ and $26 \mathrm{~ms}$, respectively), with the target's category being recognized faster when it formed a constituent with the prime (Experiment 3). In two additional experiments (Experiments 4 and 5) the authors employed not only singular primes and targets, but also plural ones. By looking at conditions with a violation at the agreement level but not at the categorical one (e.g., determiner [singular + noun $_{\text {plural }}$ ), the authors could test whether syntactic priming can be observed independently from the co-occurrence of prime and target in natural language. When participants were asked to indicate the category of the target word (Experiment 4), syntactic priming was observed under both masked and unmasked conditions, independently of agreement. However, when participants were asked to indicate the number (singular or plural) of the target, this effect disappeared (Experiment 5). In parallel, a significant priming of grammatical number emerged, both in the masked and unmasked settings. As the authors discussed, data from this experiment do not imply that categorical congruency was not analysed, but rather that it did not affect performance in this particular task and in the presence of agreement priming. Indeed, dependent variables with more dimensions that RTs (e.g., EEG and ERP measures such as the ELAN and LAN, Friederici, 2011) might be better suited for testing the interaction between categorical and number congruency in early automatic processes. Furthermore, as French plural forms tend to be longer than the singular forms (e.g., "cortège", procession, "cortèges", processions, "coopère", cooperates, "coopèrent", cooperate), it is unclear whether participants exploited low-level properties of the target words to inform their response, possibly reducing contextual effects (especially at the categorical level, which was not directly related to the task).

A recent study employing masked and unmasked priming in German provided converging evidence for effects at the abstract categorical level (Pyatigorskaya et al., 2021). Adapting the design of Berkovitch and Dehaene (2019) (Experiment 3), the authors employed the determiner ("Ein", a) and the pronoun ("Er", $h e$ ) as primes, and nouns and verbs as targets. The authors included only target nouns and verbs ending in " $\mathrm{t}$ ", which was an inflectional suffix in verbs but part of the stem for nouns (e.g., "Er kau-t", he chews, "Ein Draht- Ø", a wire). In Experiment 1, longer recognition of the target's category was obtained for ungrammatical than grammatical prime-target combinations (i.e., "* $\mathrm{Er}+$ Noun" longer than "Ein + Noun", "*Ein + Verb" longer than "Er + Verb"). Notably, this effect was reliable both with unmasked and masked presentation of the prime, albeit reduced in magnitude for the latter setting. In Experiment 2, Pyatigorskaya et al. (2021) included an additional baseline condition, in which the target words were preceded by the non-words primes "Ftn" and "Fr", matching in length and orthographic

\footnotetext{
6"Der" is both a masculine, singular, nominative form and a feminine, singular, genitive form. "Die" is both a feminine, singular, nominative form and a masculine, plural, nominative form.

${ }^{7}$ Note also that some of the female target words used by the authors share the same form for the nominative and genitive case (e.g., nominative: "die Gabel", the fork, genitive: "der Gabel”, of the fork), and some of the masculine nouns do not change the wordform between singular and plural (e.g., "der Tiger", the tiger, "die Tiger", the tigers). The authors addressed this issue in some additional analyses on a subset of items (Experiment 2), focusing on masculine nouns with different singular and plural forms.
} 
properties "Ein" and "Er" respectively. As in Experiment 1, syntactic priming was observed both in masked and unmasked setting. Furthermore, a normalization procedure (e.g., "Er kaut ${ }_{\text {NORM }}$ " = "Er kaut" - "Ft kaut") allowed to compare grammatical and ungrammatical conditions while keeping the prime constant (e.g., "Er kaut ${ }_{\text {NORM" and "Er }}$ Draht $_{\text {NORM" }}$ ") in the absence of categorical confounds 8 Automatic effects of grammaticality were observed when the prime was a pronoun (i.e., "*Er + noun ${ }_{\text {NORM" }}$ slower than

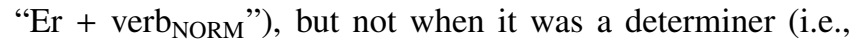
"*Ein + verb NORM" not different from "Ein + noun $_{\text {NORM"). }}$ ". Building on the model of Berkovitch and Dehaene (2019), the authors proposed that linguistic properties of the prime might influence the route to category access, according to whether it requires inflected or zero-form words to form constituents. In particular, a pronoun would point towards the recognition of category via a morphological decomposition, which occurs very fast for the verbs but turns out to be inconclusive for zero-form nouns. Conversely, a determiner in the nominative case would suggest the analysis of the upcoming word via a lemma search, which takes equal time for nouns and verbs. Indeed, in Experiment 3 Pyatigorskaya et al. (2021) showed no automatic syntactic priming when the target verbs were irregular forms, which are not morphologically decomposed. Overall, this study supports the notion that syntactic context can automatically influence how categories are accessed (Experiment 2) and that grammaticality effects rely, for some type of constituents, on morphological marking. Note that syntactic priming effects were observed in the unmasked setting in all the three experiments, possibly reflecting both automatic and late controlled processes.

Inhibition vs. facilitation. A final theoretical question of great importance is whether syntactic priming reflects a facilitatory (i.e., faster RTs driven by grammatical primetarget relationship) or inhibitory (i.e., slowing of performance driven by the violation) contextual effect. Addressing this question requires the inclusion of neutral baseline conditions, whose design is not straightforward (see Friederici and Jacobsen, 1999 for a detailed discussion). A first study (Gurjanov, Lukatela, Lukatela, et al., 1985) focusing on gender agreement in adjective-noun pairs employed pseudo-adjectives as baseline condition (Experiment 1). This study provided evidence for both facilitatory (i.e., congruent adjective-noun pairs faster than nouns preceded by pseudoadjectives) and inhibitory (i.e., incongruent adjective-noun pairs slower than nouns preceded by pseudo-adjectives) effects. However, either of these contrasts might reflect differences in lexicality of the prime (adjective versus pseudoadjective), rather than grammaticality effects (agreement versus neutral condition). Furthermore, given that the pseudoadjectives (e.g., "mej- $\varnothing$ " and "mej-a") were constructed maintaining the endings of the real-word primes (e.g., "moj$\varnothing$ ", $m y_{M A S C}$, and "moj-a", $m y_{M A S C}$ ), it remains unclear whether this conditions can be considered a neutral baseline along the gender dimension. A later study employing a similar design used a series of " $\mathrm{X}$ " as a neutral prime condition (Carello et al., 1988). This condition was included as a baseline for a separate test of associative/semantic priming (e.g., "hospital doctor" vs "XXX doctor"), and therefore not directly contrasted with grammatical or ungrammatical twoword items. However, as previously discussed (Friederici \& Jacobsen, 1999), at least at the descriptive level this study points towards inhibition, with the ungrammatical condition being $38 \mathrm{~ms}$ slower than the baseline in the lexical decision task. In studies directly testing grammatical and ungrammatical conditions against a baseline, inhibition has been reliably observed in lexical decision tasks (Schmidt, 1986) and categorical/gender categorization (Bates et al., 1996; Pyatigorskaya et al., 2021). Facilitatory effects, when present, might be limited to task and conditions relying on surfacebased processing (Bates et al., 1996, see also Friederici and Jacobsen, 1999 for a related discussion).

Interestingly, facilitation compared to a baseline has been observed at the two-word level when focusing on the semantic dimension (Lukatela, Carello, et al., 1987). This might stem from substantial difference in how incremental syntactic and semantic composition take place. At the semantic level, facilitation might reflect the pre-activation of a small set of lexical candidates (e.g., "sky", "sea") after a given word (e.g., "blue"). On the contrary, the syntactic engine might find little use in pre-activating a large set of candidates, for example all the nouns and adjectives, following the presentation of a determiner. Accordingly, the syntactic system might have proceeded with an efficient checking of incoming linguistic features and integration of their features into the previously establish context.

Additional aspects. We would like to conclude this section with some general methodological consideration regarding the studies described above. First, in most of the studies conducted in Serbo-Croatian language the participants were asked to perform a double lexical-decision task, responding to both the prime and target. Given that in most of these studies the presentation of the prime was terminated by the response of the subject, it remains unclear whether different presentation times across conditions might have affected the processing of the targets. Unfortunately, RTs to the different primes were not reported, therefore leaving this question open. Second, not all the studies provided a detailed account of how the psycholinguistic properties

\footnotetext{
${ }^{8}$ Note that in the absence of this manipulation it remains unclear whether comparing, for example, Determiner + Noun and Determiner + Verb merely reflects a categorical difference (Noun/Verb) or an actual grammaticality effect.
} 
of the stimuli employed were controlled, limiting both the reproducibility of the results and, to a certain degree, their interpretation. For example, variables such as the orthographic neighbourhood might be particularly important to control when testing lexical decision. Similarly, only a very small subset of studies reported the full stimulus list employed, making easy to replicate and extend the results under comparable conditions (see for example Goodman et al., 1981 and Seidenberg et al., 1984). Third, most of the studies conducted in Serbo-Croatian language employed pseudo-words which were created by changing a single letter from a real word. This might limit the degree to which semantic processing was carefully subtracted in these studies, possibly inflating the conclusions drawn from the pseudo-word conditions. Given the large number of relevant theoretical questions that can be addressed with this paradigm, further studies might build on these considerations to provide detailed answers to syntactic contextual effects in well-controlled settings.

Summary. Overall, the reviewed evidence from syntactic priming studies suggests that humans automatically extract abstract syntactic features from different word categories. Morphosyntactic information plays a fundamental role in driving combinatorial processing, with inhibition being observed when it results in ungrammatical combinations, rather than facilitation for correct structures.

\subsubsection{Neural indexes of syntactic violation processing: timing}

Rationale. Unless specific analysis approaches are employed (e.g., Drift Diffusion Model, Ratcliff, 1978; Ratcliff et al., 2006. Voss et al., 2004), behavioral studies provide measures such as RTs and accuracy which can vary only along a single dimension (i.e., magnitude). On the contrary, neuroimaging techniques such Electroencephalography (EEG) and Magnetoencephalography (MEG) provide multiple dimensions along which syntactic effects can be observed, such as amplitude of the responses, latency and differences in the topographical distribution (Münte \& Heinze, 1994). Furthermore, the high temporal resolution of E/MEG allows to characterize the different stages of syntactic processing, for example with a distinction between early and late components.

To the best of our knowledge, Münte, Heinze and colleagues were the first to analyse the Event-Related Potentials (ERPs, see Luck, 2014 for an introduction) elicited by syntactic violations at the two-word level (Münte \& Heinze, 1994, Münte et al., 1993). The authors were particularly interested in these basic constructions to highlight syntactic violation detection, in the absence of repairing processes which might be engaged when longer sentences are employed (see also Herrmann et al., 2012 for a similar discussion). In a first experiment conducted in German, participants read determiners or pronouns as first words, followed by a noun or an inflected verb. Three main type of conditions were included: grammatical constructions (e.g., "Ich verteidige", I defend, "Der Wurm", the MASC $_{\text {worm }}$ MASC ), agreement errors (e.g., "*Er bewundere", he admire, "*Der Haus", the MASC $_{\text {house }}$ FEM) and categorical violations (e.g., "*Der verteile", the distribute, "*Du Parlament", you parliament ${ }^{9}$. Participants performed a grammaticality judgement task, therefore actively analysing the syntactic relations between the function and content words. Compared to grammatical structures, both categorical and agreement violations resulted in an increased negativity with an anterior distribution, starting approximately $300 \mathrm{~ms}$ after the second word presentation. A similar negativity for categorical violations at the two-word level was reported in English by the same group (Münte et al., 1993), albeit with a slightly different topography possibly related to the EEG reference site (Münte \& Heinze, 1994). As we will discuss below, similar effects have been observed in the auditory modality (Hasting \& Kotz, 2008) with an earlier latency onset. Notably, at least at the agreement level 10 , there is support for the independence of syntactic negativities from semantic information and co-occurrence probabilities. In particular, increased negativities were observed for casemarked violations in adjectival phrases in Finnish using pseudo-adjectives and pseudo-nouns (Münte \& Heinze, 1994).

Morphosyntactic features. Barber and Carreiras (2003. 2005) investigated whether different types of morphosyntactic features, namely gender and number, are processed similarly by the brain. This line of research was based on conflicting findings from earlier behavioral studies (Colé \& Segui, 1994; Faussart et al., 1999; Lukatela, Kostić, Todorović, et al., 1987), and on the notion that gender but not number is a feature of the stem (Faussart et al., 1999). In a first experiment with Spanish materials (Barber \& Carreiras,

\footnotetext{
${ }^{9}$ Note that the chosen examples illustrate a feature of the experimental design, namely that each second word was presented only once to each subject. Their appearance in each of the conditions was counterbalanced across participants.

${ }^{10} \mathrm{We}$ want to point out that it might be particularly challenging (if not virtually impossible) to induce categorical violations using pseudo-words (e.g., "flirk"), as they can take any grammatical function (e.g., "they flirk", "the flirk"). Orthographic and phonological differences between grammatical categories might be exploited to construct, for example, pseudo-nouns and pseudo-verbs. Studies employing this strategy require careful designs, ensuring that grammaticality is orthogonal to low-level properties of the pseudowords (see for example the design of Hasting and Kotz, 2008, where grammaticality is orthogonal to category of both the first and second word. See also Steinhauer and Drury, 2012 for similar methodological considerations).
} 
2003), participants read two-word phrases composed of a noun and an adjective. Four conditions were included: grammatical constructions (e.g., "faro alto", lighthouse MASC SING tall $_{M A S C S I N G}$ ), gender disagreement (e.g., "*faro alta", lighthouse $_{M A S C}$ SING tall $_{\text {FEM SING }}$ ), number disagreement (e.g., "*faro altos", lighthouse ${\text { MASC SING } \operatorname{tall}_{M A S C} \text { PLUR }}_{\text {) or }}$ a double violation (e.g., "*faro altas", lighthouse MASC SING tall $\left._{F E M ~ P L U R}\right)$. Control items were included to exclude that agreement effects were driven by phonological similarity between the last vowels of nouns and adjectives. Compared to grammatical constructions, syntactic violations elicited an increased negativity between 300 and $500 \mathrm{~ms}$, with a central and centro-parietal topography (N400). Notably, the amplitude of the N400 was similar for agreement violations of gender, number and double violation. Following this timewindow, both grammatical and ungrammatical constructions elicited a positivity (P3), whose amplitude did not differ across conditions. Differences were however appreciated in the latencies of this component, which was the fastest for the grammatical condition $(536 \mathrm{~ms})$, followed by the double violation $(550 \mathrm{~ms})$, the number violation $(579 \mathrm{~ms})$ and finally by the gender disagreement $(592 \mathrm{~ms})$. Both the increased N400 for syntactic violations and the modulation of the P3 latency by the type of violation were replicated in a follow-up study (Barber \& Carreiras, 2005), which included gender and number agreement both in adjectivenoun pairs and determiner phrases (Experiment 1,Barber and Carreiras, 2005). Furthermore, an additional Left Anterior Negativity (LAN) was observed for agreement violations in determiner phrases, which was absent in adjectivenoun pairs. Interestingly, when the same determiner or adjective phrases used in Experiment 1 were included in a sentence (beginning and middle positions, respectively), violations resulted in a LAN followed by P600 (Experiment 2, Barber and Carreiras, 2005). In the context of longer sentences, differences then emerged between gender and number agreement violations, with higher amplitude of the P600b for the former condition. Overall, these two studies showed that at least the first phase of agreement analysis (LAN and N400) does not distinguish between violations of gender or number features, which are however relevant for subsequent re-analysis and repairing processes (P3 and P600). Interestingly, it seems that left-lateralisation of ERP effects depend on the presence of strong syntactic cues, either in the form of function words or a sentential context (Experiment 1 and 2, respectively in Barber and Carreiras, 2005).

Automaticity. A follow-up question which was addressed concerned the task-independence of these ERP effects. In a follow-up study, Münte and colleagues employed the same materials from their first experiment but asked the participants to perform a lexical decision task. Even when diverting the attention of the participants from the analysis of grammatical relationships, frontal negativities were observed for categorical and agreement violations (Münte \& Heinze, 1994). A similar increased negativity for syntactic violations was also observed when participants were engaged in a letter monitoring task (Munte 1992; cited in Münte and Heinze, 1994). Overall, these studies support the automatic nature of early negativities driven by syntactic violations. Converging evidence comes from a study in the auditory modality, showing an early negativity (from approximately $100 \mathrm{~ms}$ ) for agreement and categorical violations under conditions of visual distraction (Hasting \& Kotz, 2008).

Auditory modality. Compared to the reading studies reviewed above, earlier latencies have been reported for syntactic violations at the two-word level in the auditory modality. Hasting and Kotz (2008) reported an Early Syntactic Negativity (ESN) with a latency onset of approximately 100 ms for categorical and agreement violations. The ESN was elicited both when participants performed a grammaticality judgement task (Experiment 1) and when their attention was diverted away from the stimuli (Experiment 2), supporting the automaticity analysis of syntactic analysis. The presence of the ESN was replicated in two experiments with agreement (Jakuszeit et al., 2013) and categorical violations (Maran et al., 2021). Of note, Jakuszeit et al. (2013) failed to replicate the ESN effect for categorical violations, possibly due to reduced statistical power. From a methodological point of view, the earlier latency of the ESN compared to the negativities elicited in the visual modality (N400 and LAN) might reflect a more accurate time-locking of the ERP analysis (see also Hasting and Kotz, 2008 for a similar argument on the latency of the Early Left Anterior Negativity and LAN). In the visual modality it is not possible to establish at which timepoint the offending morphemes are processed, therefore the ERP analysis is usually time-locked to the word onset. Conversely, with auditory materials, Hasting and Kotz (2008) could time-lock the ERP analysis precisely at the onset of the phonemes eliciting the agreement violations (e.g., grammatical: "Du fal|test", you fold, "Er fal|tet", he folds; ungrammatical: “*Du fal|tet", you folds, "*Er fal|test", he fold, with | corresponding to the point of time-locking). A similar precision was obtained when testing categorical violations, since nouns and verbs with an ambiguous stem were used (e.g., grammatical: "Ein Fal|ter", a butterfly, "Er fal|tet", he folds; ungrammatical: "*Ein Fal|tet", a he folds, "*Er fal|ter", he butterfly). Techniques such as the crosssplicing further allowed to control for phonetic and acoustic features up to the time-locking point (Hasting \& Kotz, 2008; Hasting et al., 2007; Shtyrov et al., 2003). An interesting aspect of ESN studies (Hasting \& Kotz, 2008; Jakuszeit et al., 2013, Maran et al., 2021) is that their design, in combination with the cross-splicing technique, allows to investigate gram- 
matical effects which are orthogonal to the identity and the category of both words and to low-level of acoustic features up to the time-locking point (see Steinhauer and Drury, 2012 for a related discussion).

The ESN is not the only ERP component elicited by two-word auditory categorical or agreement violations. First, while the effect was first reported in the 100-300 ms time-window under attentive conditions (Hasting \& Kotz, 2008), follow-up studies showed a prolonged negativity lasting approximately until $500 \mathrm{~ms}$ (Jakuszeit et al., 2013, Maran et al., 2021), possibly reflecting the overlap of an ESN and a subsequent N400. This second negativity, reminiscent of the effect reported by Barber and Carreiras (2003. 2005), might reflected additional processes related to processing a mismatching suffix, as it was observed in conditions in which syntactic violations were realised with overt marking (Jakuszeit et al., 2013; Maran et al., 2021)). Second, an increased late positivity starting approximately at $500 \mathrm{~ms}$ has been reported for agreement (Hasting \& Kotz, 2008) and categorical (Jakuszeit et al., 2013; Maran et al., 2021) violations. This late positivity is elicited in conditions where the participants judge the grammaticality of items (Experiment 1 in Hasting and Kotz, 2008, Maran et al., 2021) or are not actively distracted (Jakuszeit et al., 2013). However, when care is taken to ensure that participants are not attending the auditory stimuli, only the ESN is observed (Hasting \& Kotz, 2008). In this regard, the functional profiles of the ESN and the late positivity mirror the one of the ELAN and the P600 observed with longer stimuli (Friederici, 2011; Hahne \& Friederici, 1999), suggesting that the existence of a first automatic and a second late controlled stage of analysis is a core aspect of syntactic composition.

Extra-linguistic information. We conclude this section reviewing one recent study which addressed whether extralinguistic information could influence syntactic processing at the agreement level. This study is of great interest, as it showed that grammatical two-word phrases can elicit negativities, closely resembling those elicited by agreement violations (LAN, N400), when their information does not match extra-linguistic context. Arcara et al. (2019) used a picture-phrase matching task, in which a picture representing one or multiple fruits was followed by a grammatical twoword phrase, starting with a quantifier. Relevant for the present discussion, the picture and two-word phrase could indicate a singular or multiple entities, matching indicated numerosity (e.g., the phrase "alcune mele", some apples, following the picture of four apple) or not (e.g., the same phrase following the depiction of a single apple). When the depicted numerosity and the one indicated by the phrase did not match, the noun following the quantifier elicited a negativity between 350 and $450 \mathrm{~ms}$, similar to the LAN, which is usually observed for outright agreement violations.
Interestingly, this effect was observed only in phrases denoting plural quantities, possibly implying that even within the analysis of number agreement differences might exists.

Additional aspects. In this final section, we would like to highlight some methodological aspects of the ERPs studies discussed above. As pointed out by in the context of studies employing longer constructions (Steinhauer \& Drury, 2012), sustained differences between grammatical and ungrammatical conditions before the critical word (i.e., before the second word) might be problematic, especially if combined with pre-processing steps as baseline correction. To this end, orthogonal designs such as those employed by ESN studies and baseline correction procedures based on an interval preceding the first word onset might be preferred. Furthermore, the appropriate application of common preprocessing steps such as high-pass filtering, re-referencing and baseline-correction procedure to language studies is not trivial (Maess et al., 2016, Molinaro et al., 2015, Tanner et al., 2015, Widmann et al., 2015), and should be guided by careful methodological considerations.

Summary. ERP studies employing syntactic violations provide evidence for an early automatic analysis of grammatical relationships, followed by slower task-dependent processes (Figure 3A). In particular, early negativities characterise the first stage of analysis (e.g., ESN), reflecting an all-or-none detection of grammaticality. In later time-windows (late positivity), fine-grained distinctions between the types of violations can be appreciated.

\subsubsection{Neural indexes of syntactic violation processing: lo- calization}

Rationale. Two-word syntactic violations have been used in combination with techniques with high spatial resolution (e.g., Functional Magnetic Resonance Imaging, fMRI), to highlight brain regions involved in evaluating whether the grammatical information of incoming words can be integrated into a constituent. A first fMRI reading study (Kang et al., 1999) employed well-formed verb phrases, two-word constructions being ill-formed, either due to a categorical error or to an unlikely meaning (e.g., "heard shirts"). Both syntactic and semantic violations resulted in activation of Broca's area, albeit with different patterns in its cytoarchitectonic subregions. On the one hand, Broca's area pars opercularis (Brodmann area, BA, 44) showed left-lateralised activity for both types of violations, but larger for the syntactic type. Thus, left-lateralised activity of Broca's area seems particularly relevant for the analysis of categorical information of incoming words. On the other hand, Broca's area pars triangularis (BA45) and the 
Syntactic violations: ERP and fMRI studies

A. List of studies (ERP manipulations)

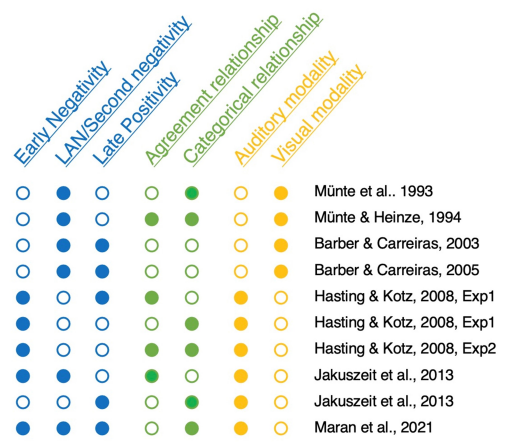

B. Categorical and agreement violations (fMRI studies)

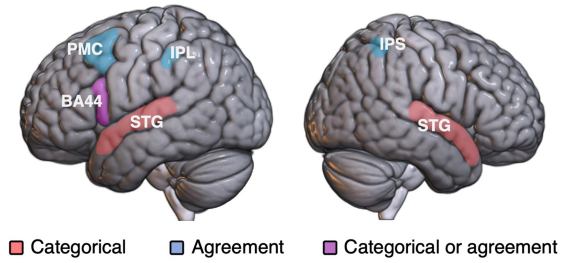

\section{Figure 3}

Summary of neuroimaging studies employing two-word syntactic violations. (A) Overview of the ERP studies; (B) Key brain regions highlighted by fMRI studies. BA44: Brodmann area 44; STG: superior temporal gyrus; PMC: premotor cortex; IPL: inferior parietal lobe; IPS: intraparietal sulcus.

more anterior portions of the left prefrontal cortex (BA46 and BA10) showed left- and right-lateralised activity for syntactic and semantic violations respectively. This study provided first evidence for a dissociation between brain regions processing syntactic and semantic information, observable even at the most minimal level of composition. However, as Kang et al. (1999) pointed out, in this study grammaticality is confounded with the category of the second word, which was always a noun in well-formed structures (e.g., "blew bubbles") and a verb in violations (e.g., "* grew heard").

Categorical violations. Adapting the original ESN paradigm (Hasting \& Kotz, 2008), Herrmann et al. (2012) contrasted auditory well-formed two-word phrases and violations at the categorical level. A German preposition (i.e., "Im", in the) or a pronoun (i.e., "Er", he) was presented as the first word, followed by an uninflected noun (e.g., "Knie- $\varnothing$ ", knee) or an inflected verb (e.g., "knie-t", kneels). The four combinations of first and second words allowed to orthogonally manipulate grammaticality and perceptual markedness of the second word (i.e., the presence of the inflectional suffix "- $t$ "). Herrmann et al. (2012) showed a clear dissociation between brain regions supporting syntactic and perceptual processes. Increased activation for syntactic violations was observed in the left IFG, in particular in BA44, and in the bilateral superior temporal gyrus (STG). Overt marking was associated with increased activity in the bilateral auditory cortices. Moreover, results from additional multivariate pattern analysis (MVPA) provided additional evidence for this dissociation. Activity patterns in left BA44, STG and posterior middle temporal gyrus (pMTG) could be used to classify grammatical and ungrammatical conditions, while bilateral activations of the primary auditory cortex could be used to discriminate between marked and unmarked items. The involvement of Broca's area in categorical analysis converges on previous fMRI data at the two-word level and points towards modality-independent computations, given that it was previously observed with visual presentation of phrases (Kang et al., 1999). On the contrary, at least at the two-word level the involvement of temporal regions in syntactic analysis seems to be restricted to the auditory modality. Due to the poor temporal resolution of fMRI, it remains underspecified which of these activations reflect the early analysis (ESN) and the later re-analysis stages (P600) discussed above.

Agreement violations. To the best of our knowledge, only 
two fMRI studies have employed agreement violations at the two-word level in comprehension. Using the same paradigm of a previous EEG study (Barber \& Carreiras, 2005), Carreiras et al. (2010) presented their participants with two-word expressions (adjective and determiner phrases) which could be well-formed or contain gender or number agreement violations. Both types of agreement violations resulted in increased activation of the left IFG and the left premotor cortex, mirroring the pattern of LAN and N400 observed in EEG studies (Barber \& Carreiras, 2003, 2005). Additionally, number violations in determiner phrases resulted in increased activity of the right parietal lobe, a region involved in numerical processing (Arcara et al., 2021, Dehaene et al., 2003, Zorzi et al., 2011). Focusing specifically on determiner phrases, Heim et al. (2010) investigated which brain regions process agreement in a modality-independent fashion. In particular, the authors presented their subjects with two-word constructions, which could be grammatical and with gender feature violations, both in the visual and auditory modality. To highlight modality-independent agreement processing, in a first step the authors created an anatomical mask, resulting from a conjunction analysis of the two modalities (i.e., brain regions which were active in both the auditory and visual presentation of the phrases). In a second step, this anatomical mask was used to restrict the results of the main effect of grammaticality to modality-independent regions only. This procedure highlighted modality-independent processing of agreement violations in the left BA44, premotor area (BA6), inferior parietal lobe and the right supplementary motor area. Overall, these studies support an involvement of the left IFG and premotor cortex in processing gender and number agreement violations. Parietal lobe activations, when present, seem to be left-lateralized for gender processing (Heim et al., 2010) and right-lateralized for violations of number (Carreiras et al., 2010).

Summary. The left IFG appears as a key region in analysing and detecting the violation of grammatical rules, both at the categorical and agreement level. These two domains seem differentially engage additional brain regions: superior and posterior middle temporal regions are activated by categorical processing, while agreement violations are processed by premotor and parietal regions (Figure 3B). Crucially, at least the involvement of the left IFG appears to be supra-modal, with activity in these regions been observed both in the auditory and visual modality.

\subsubsection{Neural indexes of syntactic violation processing: causality}

Rationale. A limitation of neuro-imaging techniques is that they allow to test only a correlational link between brain activity and a given linguistic process of interest. To overcome this limitation, two complementary approaches have been employed. First, neuroimaging and behavioral data have been acquired from patients with specific brain lesions (Vaidya et al., 2019). Second, neurostimulation techniques such as Transcranial Magnetic Stimulation (TMS) have been employed to temporarily disrupt brain functioning in healthy participants engaged in specific tasks of interest (Hartwigsen, 2015), possibly in combinations with neuroimaging techniques. The rationale of both approaches is that, if a brain region is necessary for a given process of interest, its permanent damage (lesion studies) or transient perturbation (TMS studies) should affect the behavioral or neural correlates of the operation of interest.

Cortical lesions. Patient and neurostimulations studies employing two-word syntactic violations have focused on Broca's area, given the fMRI studies described above. In a follow-up study employing the ESN paradigm, Jakuszeit et al. (2013) compared healthy controls and patients with left IFG lesion, to test the causal role of this region in generating top-down categorical and morphological predictions. The presence of top-down syntactic predictions, generated from the left IFG, could in principle explain the earliness of violations effects observed in ERP studies (ELAN, Friederici et al., 1993; ESN, Hasting and Kotz, 2008). When comparing well-formed pronoun-verb combinations and agreement violations, an ESN (100-300 ms) was evident in healthy controls but absent in patients with a left IFG lesion. Both groups showed a subsequent posterior negativity, between 300 and $500 \mathrm{~ms}$. In this study, categorical violations failed to elicit a significant ESN effect in both controls and patients, possibly due to the reduced power compared to the original study of Hasting and Kotz (2008). However differences were observed in a later time-window (550-900 $\mathrm{ms}$ ), with the presence of a P600 effect in controls but not in patients. Jakuszeit et al. (2013) suggest that the pattern of results is compatible with a deficit in serial predictive mechanisms, engaged by agreement but not hierarchical categorical levels, as the same patients showed a lack of an $\mathrm{N} 2 \mathrm{~b}$ response in an additional oddball paradigm. Note that, as previously pointed out (Maran et al., 2021), the lack of an ESN is also compatible with a role of the left IFG in detecting an agreement violation, as by definition this region in patients is damaged both during the prediction and integration phase. The absence of a late positivity for categorical violations is compatible with a causal role of the left IFG in repairing or re-analysis processes when a minimal syntactic context is available. As an intact P600 was previously observed in Broca's patients for categorical violations in longer and more complex structures (Friederici, Von Cramon, et al., 1999), it remains an open question under which circumstances repairing and re-analysis processes can 
be initiated despite damage of the left IFG. Importantly, the small number of patients and healthy controls included in the study of Jakuszeit et al. (2013) (10 per group) might have made the study underpowered, especially for detecting early categorical effects, where two types of constituents (determiner phrases and sentences) are present in each level of grammaticality.

Neurostimulation. The causal role of the left IFG in generating categorical predictions has been recently tested in a combined EEG-TMS study (Maran et al., 2021). In this study, the authors employed an ESN paradigm with categorical violations, with determiner and pronouns being followed by nouns or verbs. Using online TMS ${ }^{11}$ Broca's area functioning was disrupted specifically at the predictive stage of a two-word phrase (i.e., during a determiner predicting a noun). Compared to lesion studies, this approach has the advantage of inducing a temporally specific interference, targeting a specific cognitive process. Interestingly, the authors reported a prolonged early negativity effect $(200-450 \mathrm{~ms})$, possibly reflecting the overlap of an ESN and a second posterior effect, followed by a late positivity. However, these components were not affected by Broca's area stimulation at the predictive stage. Even at a more fine-grained level of analysis, with an accurate modelling of the TMS-induced electrical field (Numssen et al., 2021; Weise et al., 2020), no effect of Broca's area disruption during the predictive phase could be observed. As the authors suggested, these findings do not support a causal role of Broca's area in generating syntactic predictions at the categorical level, but are compatible with a role of this region in the bottom-up integration of words into constituents. This remains a testable hypothesis for future studies. Indeed, a previous study found an effect of TMS over the left IFG during the second word (Sakai et al., 2002), when focusing on violations of verb transitivity in basic twoword construction. Faster RTs in judging the grammaticality of the items were observed when TMS occurred over the left IFG $150 \mathrm{~ms}$ after the second word (verb) presentation. Note however that also this study has a very limited sample size $(\mathrm{N}=6)$.

The causal involvement of Broca's area in syntactic processing, and specifically in agreement analysis, has been further tested by an online TMS behavioral study (Carreiras et al., 2012). In this study, participants performed a grammaticality judgement task on determiner phrases, which contained agreement violations or were well-formed. Broca's area and the right intraparietal sulcus, with the same coordinates as the activations reported in a previous fMRI study (Carreiras et al., 2010), served as targets (experimental and control site, respectively). The effect of TMS on each site was compared against a condition in which no stimulation occurred. Replicating previous findings (Carreiras et al., 2010), faster RTs were observed for grammatical than ungrammatical items. Crucially, this difference was selectively reduced by TMS over Broca's. This study therefore provides initial evidence for the causal involvement of Broca's area in morphosyntactic processing, and specifically in syntactic checking and integration of features, given that TMS was delivered during the second word of a constituent. In light of this study, the absence of an ESN for agreement violations in patients with left IFG lesions (Jakuszeit et al., 2013) could also reflect a deficit in bottom-up integration rather than top-down prediction.

Summary. Patient and TMS data support a causal involvement of Broca's area in processing syntactic violations (Carreiras et al., 2012; Jakuszeit et al., 2013). From a parsing perspective, the available evidence supports a role of this region in bottom-up integration (Carreiras et al., 2012) rather than prediction (Maran et al., 2021).

\subsubsection{Syntactic violations: syntactic Mismatch Negativ- ity (sMMN)}

Rationale. Syntactic Mismatch Negativity (sMMN) studies employ an adapted version of the classic oddball paradigm, in which a rarely presented deviant stimulus occurs among repeated standard stimuli. In its traditional form, deviant and standard stimuli are presented acoustically to the subjects, who are distracted by means of a silent movie. A classic ERP finding is that an increased response (MMN) is observed for the deviant stimuli compared to the standard ones (Näätänen et al., 1978). Traditional MMN paradigms have focused on central auditory processing, as standard and deviant stimuli usually differ along a dimension of interest such as frequency and intensity (for a review see Näätänen et al., 2007). In its sMMN form, a grammatical (e.g., "we come") and an ungrammatical (e.g., "*we comes) construction are used as stimuli, being employed both as a standard and as a deviant in the oddball (Figure 4). For example, in a first block "we come" and "*we comes" are used as standard and deviant, respectively. In a second block, the opposite assignment is employed, taking care to counterbalance the order of the blocks across subjects (Pulvermüller \& Shtyrov, 2003). The sMMN effect consists in a larger MMN response evoked by ungrammatical deviants compared to grammatical ones (Figure 4), usually observed with a latency smaller than $200 \mathrm{~ms}$ (Pulvermüller \& Shtyrov, 2003, Shtyrov et al., 2003). This effect stems from automatic linguistic processing, as similarly to the classic MMN studies participants are usually actively distracted by means of silent movies.

\footnotetext{
${ }^{11}$ The term online TMS is used to refer to the delivery of TMS pulses during the task, as opposed to offline TMS where after-effects of the stimulations are used to induce a virtual lesion (for a review, see Hartwigsen, 2015).
} 


\section{Syntactic Mismatch Negativity (sMMN)}

A. List of studies

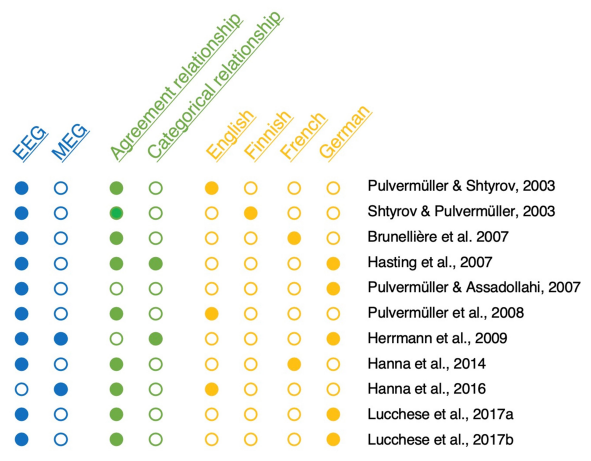

B. Standard experimental paradigm

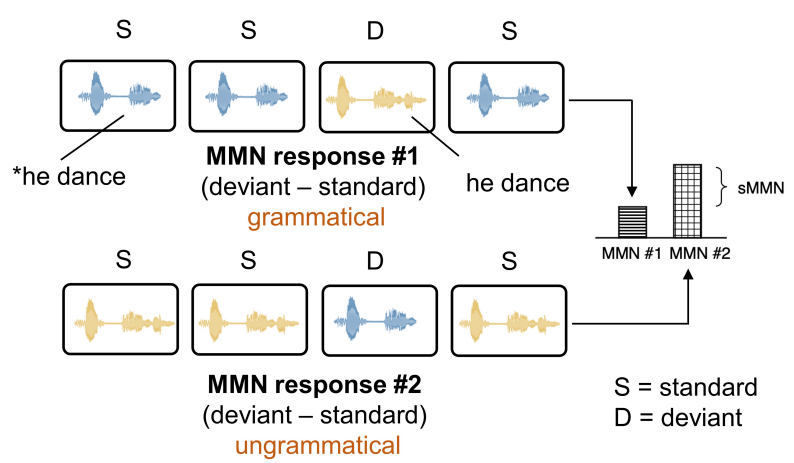

\section{Figure 4}

Summary of the sMMN studies. (A) List of studies, with an overview of the included manipulations; (B) Example of a standard sMMN paradigm.

In sMMN paradigms, great care is taken to control for acoustic features of the stimuli. Procedures such as the crosssplicing technique make the different items acoustically identical up to the information which makes trials grammatical or ungrammatical (Pulvermüller \& Shtyrov, 2003). Furthermore, control conditions are included to ensure that the sMMN depends on grammaticality and is not confounded by acoustic features (e.g., the presence of the "-s" suffix in the examples above). In this regard two strategies are usually employed. Additional blocks can be included, in which the two second words are presented out of context (e.g., "[noise] come", "[noise] comes), both as deviant and standards. The MMN response to these stimuli can be subtracted from the respective two-word utterances (e.g. "we comes" - "[noise] comes" and "*we comes" - "[noise] comes"), therefore allowing to control for acoustic differences between grammatical and ungrammatical items (Shtyrov et al., 2003). A second strategy consists in using two-word constructions which make grammaticality orthogonal to the suffix and first word (e.g., "Mä tuon", I bring IST PERSON SINGULAR, "*Mä tuot", I bring 2 ND PERSON SINGULAR, "*Sä tuon", you bring IST PERSON SINGULAR, "Sä tuot", you bring $_{2 N D}$ PERSON SINGULAR, from the Finnish materials in Shtyrov et al., 2003). From a methodological point of view, the use of only few two-word items in each study (e.g., one correct and one incorrect) might allow to characterise very early stages of linguistic analysis, which might be lost in the ERP averaging process if stimulus materials variance results in jittered evoked activity (Pulvermüller \& Shtyrov, 2003). At the same time, generalizability of the results needs to be ensured by varying the stimulus lists across studies.

Automaticity. As in the case of attended syntactic violations, sMMN paradigms have mostly focused on agreement and categorical features. At the agreement level, sMMN effects have been reported in several languages: English (Hanna et al., 2016; Pulvermüller \& Shtyrov, 2003), Finnish (Shtyrov et al., 2003)), French (Brunellière et al., 2007; Hanna et al., 2014) and German (Hasting et al., 2007; Lucchese, Hanna, et al., 2017; Lucchese, Pulvermüller, et al., 2017; Pulvermüller \& Assadollahi, 2007). These studies converge in reporting an automatic effect of grammaticality, with ungrammatical two-word utterances used as deviants eliciting a more negative ${ }^{12}$ MMN that the correct counterparts, generally occurring before $200 \mathrm{~ms}$. Similar effects have been reported

\footnotetext{
${ }^{12}$ In one study (Lucchese, Hanna, et al., 2017) an effect of the opposite polarity was observed, possibly due to the short SOA used by the authors.
} 
in sMMN studies employing violation at the categorical level (Hasting et al., 2007; Herrmann et al., 2009). From a cognitive point of view, the sMMN effect has been described as a neurophysiological trace of syntactic priming (Hasting et al., 2007, Pulvermüller \& Shtyrov, 2003). At least at the agreement level, this effect has been linked to facilitation for grammatical structures rather than the result of violation detection, as no difference was observed between the MMN evoked by ungrammatical deviants and words presented following noise (Pulvermüller \& Shtyrov, 2003, 2006). It is unclear whether this explanation applies to categorical sMMN effects, as a recent syntactic priming study including a baseline condition reported only inhibition effects for ungrammatical constructions (Pyatigorskaya et al., 2021). An interesting hypothesis is that these diverging findings might reflect a difference between the processing of agreement features (Pulvermüller \& Shtyrov, 2003) and categorical information (Pyatigorskaya et al., 2021). In this regard, it has been pointed out that the sMMN for agreement and categorical violations have different topographies (Hasting et al., 2007)), possibly reflecting separate underlying processes.

Rule-based analysis. One potential issue which arises when comparing grammatical and ungrammatical expressions is that these conditions differ not only at the syntactic level, but also in the frequency of occurrence, which is virtually zero for violations. Accordingly, the sMMN and the results from the studies employing syntactic violations reviewed above might reflect either a grammatical process or purely differences in transition probabilities. Two carefully designed sMMN experiments addressed this issue, supporting a purely grammatical nature of the processes reflected in this ERP component. The rationale of these two sMMN studies (Herrmann et al., 2009, Pulvermüller \& Assadollahi, 2007) on the categorical and agreement features respectively, is very similar. In both studies, together with grammatical (frequent) and ungrammatical two-word expression, rare grammatical constructions were used. The authors hypothesised that, if the sMMN reflects the application of grammar, the MMN evoked by rare and common grammatical constructions would be similar and smaller than syntactic violations. This is exactly what was observed in both studies, providing evidence for a purely grammatical explanation of the reported effects.

Seriality vs. interactivity. Studies employing basic sMMN paradigms support the notion that syntactic analysis occurs in an automatic fashion. Building on these findings, a recent multi-feature sMMN paradigm (see Näätänen et al., 2004 for a description) sought to investigate whether lexicosemantic and syntactic processing would occur strictly in a serial fashion or would interact in early stages of processing (Lucchese, Hanna, et al., 2017). In this study, two dimensions of the utterances were manipulated, namely the lexicality of the second word (real verb or pseudo-verb) and the grammaticality of agreement relationship between pronoun and second word. Thus, four conditions were included: well-formed expressions (agreement between pronoun and verb), grammatical but with a violation of lexicality (agreement between pronoun and pseudo-verb), ungrammatical but lexical (pronoun and verb, but with agreement violation) and a double violation (pronoun and pseudo-verb, with an agreement violation). This study reported an early (70-210 ms) interaction between lexicality and agreement analysis, challenging serial processing of linguistic information. While a clear sMMN effect was observed for the double violation, the fact that the MMN of grammatical items and agreement violations realised with real verbs were not statistically different is somehow difficult to interpret. In particular, at least in the very early phase of the sMMN effect (70-140 ms) the amplitude for this condition does not significantly differ from any of the other conditions. One possibility is that this pattern of results depends on the multi-feature nature of the sMMN paradigm employed by the authors. Given the relevance of this research question for psycholinguistic models of language comprehension, future replications of this study might provide clear insights into the early interaction between lexical access and agreement feature analysis. The syntactic priming studies employing inflected pseudo-words reviewed above can further provide a behavioral background to this line of research.

Neural generators. With respect to the neural generators of the sMMN, the available evidence points towards an involvement of the left superior temporal lobe (Hanna et al., 2016. Herrmann et al., 2009, Shtyrov et al., 2003) and frontal (Pulvermüller \& Shtyrov, 2003) regions, with one study highlighting Broca's area in particular (Hanna et al., 2014). Additionally sources in the primary auditory cortex have also been reported in one study (Herrmann et al., 2009), in which the sMMN effect was observed only in the presence of overt marking of the category. At present it is still unclear why no consistent source localization of the sMMN is present in the literature. Some authors have suggested that different results might depend on the neuroimaging techniques used (Herrmann et al., 2009, Pulvermüller \& Shtyrov, 2006), as the sMMN was localised in the left frontal cortex in EEG studies (Hanna et al., 2014, Pulvermüller \& Shtyrov, 2003), while involvement of the temporal lobe was shown with MEG recordings (Hanna et al., 2016; Herrmann et al., 2009; Shtyrov et al., 2003). The diverging findings might reflect the different sensitivity of EEG and MEG to radial and tangential dipoles, or to distortions inherent to the source localization procedure (Baillet, 2017). Thus, at present further evidence is needed to draw more precise 
conclusions on the neural generators of the sMMN. Studies acquiring simultaneous EEG and MEG recordings might provide crucial insights on the localization debate (Baillet, 2017).

Additional aspects. While the reviewed studies highlighted the relevance of the sMMN for characterising syntactic processes, this paradigm might also have important clinical applications. In particular, the sMMN might allow to avoid both an under- and an overestimation of linguistic functioning, which would otherwise interfere both with diagnosis and treatment evaluation. On the one hand, this paradigm ensures that non-specific effects of lesions or conditions (e.g., fatigue or lack of sustained attention) lead to an underestimation of patients' functioning, given that no task is employed. On the other, the lack of a task might allow to evaluate the treatment outcome, linking it specifically to linguistic abilities. This is of particular importance as at later stages of recovery task-related and strategic processes might become available to patients and lead to an overestimation of their language abilities (Lucchese, Pulvermüller, et al., 2017). Recently, a sMMN multi-feature paradigm has been used to assess the outcome of speech language therapy in patients with post-stroke aphasia (Lucchese, Pulvermüller, et al., 2017). In this study, MMN was recorded employing a grammatical two-word expression (pronoun and verb), a grammatical but not lexical phrases (pronoun and correctly inflected pseudo-verb) and agreement violations. Patients were tested before and after four weeks of intensive training. Treatment selectively affected the MMN evoked by grammatical constructions and correctly inflected pseudo-word phrases. This finding nicely fits with the observation that the ungrammatical constructions and words out of context elicit comparable MMN (Pulvermüller \& Shtyrov, 2003), possibly suggesting that speech therapy began to restore the functionality of the syntactic combinatorial mechanism. However, considering the small sample size $(\mathrm{N}=10)$ and the lack of a significant correlation between the changes in the MMN amplitude and standardised clinical inventories of language abilities, further studies are needed to draw stronger conclusions. Furthermore, the lack of a healthy control group and analysis based on the grammaticality of the deviant do not allow to estimate whether a proper sMMN effect was restored following speech therapy.

A further application of the sMMN paradigm is the study of the neural basis of second language acquisition. In this context, the sMMN is an idea tool to quantify rapid and automatic syntactic processes, as the ones supporting everyday language use(Hanna et al., 2016). Furthermore, considering that only few stimuli are used standards and deviants, it is possible to test grammatical knowledge even in learners who might have a small vocabulary of the second language. In a two-word study focusing on agreement features,Hanna et al. (2016) compared the sMMN response of English native speakers and non-native learners with different levels of proficiency. Interestingly, comparable sMMN were observed for native speakers and learners with high-level of proficiency, in both cases larger than the non-proficient group. Furthermore, sMMN effects were localised in bilateral superior temporal lobes, and significant correlations were found between source-level activity and behavioral measures of syntactic abilities. Thus, these finding supports the notion that second language analysis might be supported by early and automatic mechanisms, similar to those of the first language.

We conclude this section by pointing out that, as in some studies employing attended syntactic violations (Barber \& Carreiras, 2003, 2005, Jakuszeit et al., 2013, Maran et al., 2021), some sMMN studies reported a second negativity following the early effect of grammaticality (Hanna et al., 2014, Hasting et al., 2007; Herrmann et al., 2009)). As we have previously proposed (Maran et al., 2021), this negativity could reflect to the detection of a violation between an expected suffix and the current one by heard. The presence of this effect in sMMN paradigms provides initial evidence for an automatic nature of its underlying cognitive process.

Summary. Studies employing the sMMN paradigm have provided evidence for automatic rule-based syntactic processes, independent of transition probabilities (Herrmann et al., 2009, Pulvermüller \& Assadollahi, 2007). While the available evidence points towards neural generators in the fronto-temporal cortices, further studies are needed to characterise precisely the sources of these component.

\subsection{Phrases and sentences versus lists}

Rationale. The rationale of the phrase/sentence-versus-list paradigm is simple: phrases (e.g., a determiner phrase) are characterised by hierarchical dependencies between a head (e.g., a determiner) and a dependent (e.g., a noun), while lists (e.g., a noun followed by a noun) are not. Therefore, this paradigm can be used to highlight the neural correlates of hierarchical processing in language. In principle, hierarchical processing can be tested comparing sentences and lists beyond the two-word level, an approach employed by many fMRI studies (Goucha \& Friederici, 2015; Matchin et al., 2017, Snijders et al., 2010, Zaccarella, Meyer, et al., 2017) and MEG studies (Hultén et al., 2019, Lam et al., 2016, Schoffelen et al., 2017). However, unless careful control conditions are included (Goucha \& Friederici, 2015; Pallier et al., 2011), contrasting sentences and lists does not highlight specifically syntactic composition, since in the former type of stimuli both hierarchical structure and sentential meaning are built. Furthermore, long sentence stimuli differ from lists not only because of the presence of a hierarchical structure, but 
also because of "progression effects". In particular, words which are part of a sentence, especially in late positions, benefit from contextual effects which are absent in the lists conditions and have been linked to specific neurophysiological correlates (Hultén et al., 2019; Lam et al., 2016). Thus, by highlighting the single analysis of the head-dependent relationship, two-word constructions provide an ideal level of observation of hierarchical processing.

We discuss in this section also a recent MEG study conducted in Standard Arabic language (Matar et al., 2021)), which tested hierarchical processing by manipulating syntactic complexity in semantically matched two-word expressions. This study did not include a list condition, as three different structures were compared: indefinite phrase, definite phrase, full sentence. However, the comparison of syntactic trees of increased complexity is related to contrasting structures with and without a hierarchical structure.

\subsubsection{Phrases and sentences versus lists: neurophysiolog- ical correlates}

EEG and MEG studies employing the phrase-versus-list paradigm mostly focused on the oscillatory dynamics of syntactic processing, rather than its ERP correlates. This allowed researchers to interpret their findings in light of a rich literature, addressing how specific rhythms can be well-suited for distinct linguistic computations (for reviews see: Benítez-Burraco and Murphy, 2019, Lewis et al., 2015, Meyer, 2018, Murphy, 2015; Weiss and Mueller, 2012). This approach complements and extends the ERP literature on syntactic processing, mostly based on the use of violations (Friederici, 2011). Furthermore, it provides the possibility to understand the neural basis of syntactic composition under primary principles governing brain functioning (Buzsáki \& Draguhn, 2004; Friederici \& Singer, 2015; Fries, 2015).

Alpha and beta oscillations. A first study by Segaert et al. (2018) points towards a role of alpha and beta oscillations in syntactic binding. In this study, Dutch two-word items were presented auditorily to the subjects, with a pseudo-verb following either a personal pronoun or another pseudo-verb. By comparing the two-word sentence against the list of two pseudo-verbs, the authors highlighted the neurophysiological correlates of syntactic composition in the absence of semantic information. Three main findings were reported by the authors. First, compared to the list condition, increased synchronization in the alpha and beta bands were observed in the pseudo-sentence condition, already before the onset of the second word. Second, following the second word, increased synchronization in the alpha band was found for the pseudo-sentence condition. Finally, additional analysis on the P600 component showed no significant differences between the two conditions. Importantly, given the use of minimal structures and pseudo-verbs, the oscillatory changes should be interpreted as markers of syntactic composition, as working memory demands and semantic information were minimal.

A role of beta-band in hierarchical processing is also supported by a recent MEG study conducted in Standard Arabic language (Matar et al., 2021), comparing two-word constituents of different complexity. In this study, the authors employed three structures of increasing complexity (i.e., simple indefinite phrases, definite phrases, and sentences), via the inclusion of a define article (i.e., indefinite noun and indefinite adjective, definite noun and definite adjective, definite noun, and indefinite adjective, respectively). Crucially, as in Standard Arabic the definite article is not separated by the adjective and nouns, the authors could keep the number of words equal across conditions. Increased synchronization in the beta-band following the second word was observed for more complex two-word constituents, suggesting a role of this frequency band in hierarchical processing.

Age-related changes. A recent study conducted in English, adapting the paradigm of Segaert et al. (2018), tested whether differences in the neural correlates of syntactic binding could be observed when comparing older and young adults (Poulisse et al., 2020). This study builds on previous evidence that, especially in the absence of semantic information, older population show impairment in processing basic two-word constructions (Poulisse et al., 2019). Older adults showed increased synchronization in the theta, alpha and beta bands for the list condition compared to the pseudo-sentence. This effect was observed across the two-words, being particularly sustained for the alpha band. Data from young adults showed no significant difference between the conditions, albeit a trend to increased synchronization for the pseudo-sentence condition in the alpha band was present following the second word onset. The lack of statistical significance might be due to reduced power of the follow-up study, which included approximately $65 \%$ of the trials per condition compared to the first study by Segaert et al. (2018). Significant differences between groups in the syntactic binding effect (pseudo-sentence versus list) were appreciated in the alpha band, reflecting the trend present in the young adults and the effect in the opposite direction observed in the older adults. It must be noted that in this study significant differences between pseudo-sentences and lists were also observed in the timeand phase-locked ERPs, with increased positivity in the N1, P1 and P600 components.

Summary. At present only a very limited number of EEG and MEG studies employed the phrase-versus-list paradigm, therefore further studies are needed. Preliminary evidence exists for a role of alpha and beta band synchronization in 
Phrase/sentence vs. list processing: E/MEG and fMRI studies

A. List of studies (neural oscillations E/MEG)

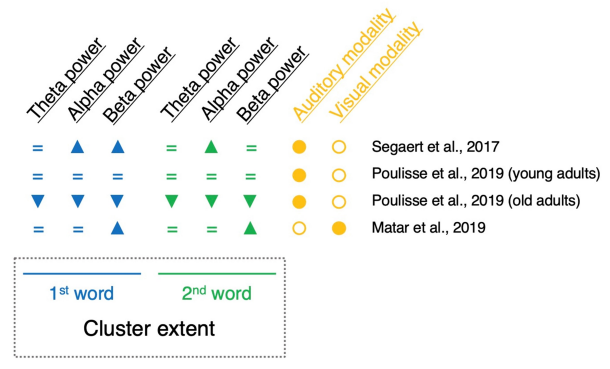

B. Localization and functional connectivity (fMRI, MEG studies)

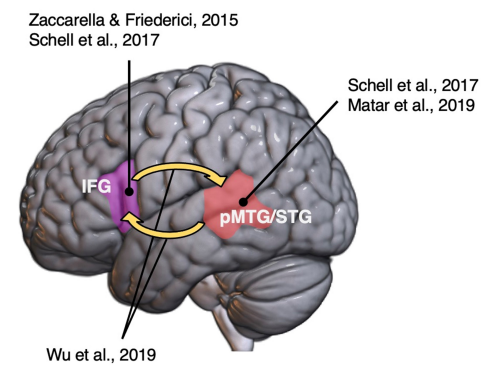

\section{Figure 5}

Summary of studies comparing phrases and sentences against lists. (A) List of E/MEG studies, summarizing the changes (increase, decrease, absent) observed in oscillatory synchronization; (B) Key brain regions highlighted by fMRI and MEG studies. IFG: Inferior frontal gyrus; pMTG: posterior middle temporal gyrus; pSTG: posterior superior temporal gyrus.

syntactic binding, and in its age-related changes (Figure 5A). It remains still unclear whether traditional ERP techniques can highlight this process, possibly due to the lack of an overt violation.

\subsubsection{Phrases and sentences versus lists: functional local- ization}

The cortical syntactic network. The comparison of phrases versus lists has highlighted the left IFG and the posterior temporal lobe as key regions involved in hierarchical processing. A first fMRI study by Zaccarella and Friederici (2015a) contrasted determiner phrases including a German pseudonoun (e.g., "Diese Flirk", this flirk) and noun-pseudo-noun lists (e.g., "Apfel Flirk", apple flirk). Additional one-word conditions were presented, including a determiner or a noun followed by a non-linguistic character string (e.g., "Diese", this, and "Apfel ", apple ). Increased activity was found in the ventral-anterior portion of BA44 for two-word phrases, which was the only condition with hierarchical dependency between the linguistic units. Conversely, evolutionary older brain regions, such as the frontal operculum (FOP) and anterior insula (aINS), reflected mere accumulation of words, with increased activation for both two-word phrases and lists compared to single words (Zaccarella \& Friederici, 2015a, 2015b). At the two-word level, activity of the left IFG might be amplified by merging operations involving a closedclass and an open-class element. In a follow-up fMRI study Schell et al. (2017), participants were presented with a German noun in isolation or in two phrasal context, following either a determiner or an adjective. Compared to single words, both types of phrases resulted in increased activation of the left Broca's area, albeit with different patterns. Phrases including a closed-class element activated specifically Broca's area pars opercularis (BA44), in its ventralanterior portion, while increased activation in the pars triangularis (BA45) was found for the combination of an adjective and a noun. Additional activations for the determiner phrase were observed in the posterior middle temporal gyrus (pMTG) and superior temporal sulcus (STS). Direct comparison of the determiner phrase against the adjective-noun pair revealed increased activity in the ventral portion of the left IFG and posterior temporal regions. A link between merging by closed-class elements and activity in BA44 was further corroborated by a conjunction analysis, highlighting the creation of a constituent specially with a function word. Inter- 
estingly, these results do not fully converge on previous data obtained in English (Bozic et al., 2015). In this fMRI study noun and verbs could be presented as stems (e.g., "rug"), inflected forms (e.g., "rugs") or two-word phrases or sentences (e.g., "a rug"). The authors showed that, while activity in the left IFG and posterior temporal lobe differentiated inflected forms for the other conditions, phrasal processing was captured by bilateral patterns of activity in the superior and middle temporal gyri. A possible explanation of the diverging results might be found in the different role that German and English assign to rule-based composition and linear order.

A recent fMRI study (Wu et al., 2019) investigated whether an involvement of the left IFG in phrase construction could be observed in Chinese, a language whose comprehension might more strongly on semantic information ( $\mathrm{Yu}$ \& Zhang, 2008; Zhang et al., 2010). In a paradigm similar to the one of (Zaccarella \& Friederici, 2015a), the authors employed two-word phrases (e.g., "this" and a classifier) and lists (e.g., two classifiers), or a single determiner or classifier. The results of the study with Chinese materials differed from those reported in German in three main aspects. First, no brain region showed increased activation specifically for two-word starting with a determiner. Broca's area (BA44 and 45 ) and the anterior insula rather responded to the presence of two-word items. Secondly, the presence of a determiner at the beginning of a two-word construction or in isolation engaged activity in BA45 and posterior temporal regions, which therefore responded to the opening of a determiner phrase. Finally, an involvement of BA44 in phrasal building was observed in directed connectivity analysis, with increased transmission of information from this region to the pMTG for conditions starting with a determiner. Increased backwards connections were observed for the conditions in which either a phrase was opened but not closed (i.e., a single determiner) or not opened at all (i.e., two classifiers), possibly signalling a failed attempt at incremental composition. An involvement of the posterior temporal lobe in hierarchical processing is also supported by the recent MEG study with Standard Arabic noun-adjective pairs (Matar et al., 2021), briefly introduced at the beginning of this section. Activity in the posterior temporal lobe was modulated by the degree of syntactic complexity of the constituent.

Indirect comparison of two-word phrases and lists can be found in some additional studies employing longer materials. Matchin et al. (2017) presented their participants six words, either as a full sentence (e.g., "the poet will recite a verse"), as three two-word phrases (e.g., "the fencer the baby their bill") or as a list ("rabbit the could extract catch protect"). In this study, no difference was found between phrases and lists. However, the list condition included closed-class elements, on which the subjects might have performed automatic chunking or repairing P600-releated processes (Friederici, 2011), based on available function words (e.g., "rabbit the" $\rightarrow$ "the rabbit"). Therefore, phrase-related composition might have been cancelled out. In a study conducted in French, Pallier et al. (2011) presented their participants constituents of different size (from 1 to 12 words). Left IFG and pSTG showed, overall, an increase of activity mirroring the increase size of the constituent, irrespective of the presence or absence of semantic information. Descriptively, a difference between the constituent of size 2 and 1 can already be appreciated in the left pSTG. Similar results were obtained in French sign language (Moreno et al., 2018), together with a larger number of cortical and sub-cortical structures, possibly reflecting modality-dependent processes or the presence of semantic information retained in the stimuli.

Differences with the red-boat studies. We have not discussed in the present section the so-called "red boat" studies (e.g., Bemis and Pylkkänen, 2011, given that a well-structured research program has highlighted the semantic and conceptual nature of this task (Pylkkänen, 2019, 2020). Studies employing the "red boat" paradigm contrast two-word and one-word expressions, in compositional (e.g., "red boat" vs "xtp boat") and list (e.g., "cup boat" vs "xtp boat") contexts. Across multiple studies, activation of the left ATL and ventromedial prefrontal cortex (vmPFC) for two-word expressions was observed only in the compositional context (and the respective task). However, given that adjective-noun form a constituent, the absence of effects in the left IFG and posterior temporal lobe is somehow unexpected and deserve careful consideration.

A first possible reason for the null effects in these regions is the lack of function words in the adjective-noun pairs, as reliable activation of the left IFG (Schell et al., 2017, Wu et al., 2019; Zaccarella \& Friederici, 2015a) and posterior temporal regions (Matar et al., 2021, Schell et al., 2017) is found when constituents include closedclass elements. Secondly, variability in the time-course of syntactic composition might make this process more elusive to techniques with high-temporal resolution such as MEG and EEG (Kochari et al., 2021), especially in the absence of an overt violation. This argument applies in particular to activations of the left IFG, as syntax-related activity in the posterior temporal lobe was found in the MEG study of Matar et al. (2021). Similarly, activity in left IFG might be more difficult to capture with MEG, due to its anatomical position and the reduced coverage from the helmet sensors. As previously pointed out (Kochari et al., 2021), the use of structural templates rather than individual MRIs might also have resulted in a minor sensitivity to changes of activity in this region in some of the "red boat" studies, especially given the precise localization of composition effects in BA44's clusters (Zaccarella \& Friederici, 2015a). Thirdly, it is important to consider that building minimal syntactic 
structures might rely on synchronized oscillatory activity (see previous section) which is not necessary phase-locked, contrary to the one highlighted in the "red boat" studies. Fourth, it is possible that no effects were observed in these two regions because in the red-boat English studies the adjective (e.g., "red") could also be interpreted as a noun (e.g., the colour red), resulting in a noun-noun list. Indeed, in languages where the presence of inflectional morphology removes ambiguity regarding the category of the first word, increased activation is observed in the left IFG for phrases composed of an adjective and a noun (e.g., Blaues Schiff, blue ship) compared to single words (Schell et al., 2017). Finally, two studies employing the "red boat" paradigm (Fló et al., 2020, Neufeld et al., 2016) have also shown that the neural correlates highlighted might, under certain circumstances, reflect task-progression or expectancy effects rather than compositional processes. Experimental designs dissociating explicit and implicit semantic processing at the phrasal level (Graessner, Zaccarella, \& Hartwigsen, 2021) might provide useful insights onto task-dependency of the conceptual-semantic network activation.

Summary. The left IFG and pMTG/STG (Figure 5B) appear as key regions in combining categorical information in hierarchical structures (Matar et al., 2021, Schell et al., 2017, Wu et al., 2019; Zaccarella \& Friederici, 2015a). Further studies are needed to address whether activations of these regions vary cross-linguistically, possibly according role of serial order in different languages (Bozic et al.,2015). Importantly, recent data support the notion that hierarchical structures might be built not only via increased activation of left IFG and PTL, but also with increased directed connectivity between these regions (Wu et al., 2019).

\subsection{Comparison of nouns and verbs in two-word constructions}

Rationale. In the previous sections we have addressed the neural basis of syntactic composition, either with the use of violations or with the comparison of constituents and lists. Especially when focusing on phrasal building effects, an underlying assumption is the existence of neuro-cognitive operations to extract categorical information from incoming words. In the present section we review a small number of studies which employed basic two-word constructions to highlight the neural basis distinction of nouns and verbs categorical representations. Importantly, despite being one of the most basic distinctions found human languages, isolating their different neural substrates remains challenging (Crepaldi et al., 2013, Crepaldi et al., 2011; Vigliocco et al., 2011), given that these two categories differ along several syntactic and semantic dimensions. Two-word constructions might provide useful insights into this aspect, as they repre- sent the most minimal level at which the syntactic features of these categories are engaged, at least in non pro-drop languages. Most of these studies share the assumptions that in order to observe traces of categorical differences, nouns and verbs need to be part of constructions where their syntactic features are necessarily extracted (see Vigliocco et al., 2011. for a similar discussion on combinatorial theories of category representation). Their results should be considered in the broader theoretical discussion on noun and verb differences (Crepaldi et al., 2011, Vigliocco et al., 2011). Importantly, two-word constructions formed with nouns and verb are, in most of the cases, two different types of constituents, namely phrases and sentences. Similarly, thematic role assignment might be differently engaged by these constructions, unless specific types of phrases are employed (Zaccarella, Meyer, et al., 2017).

A first study conducted by Fiebach et al. (2002) in German supports the hypothesis that differences between noun and verbs might emerge specifically when they are part of minimal phrases or sentences. In this study, participants were presented with noun and verbs, matched for acoustic properties, in isolation or preceded respectively by a determiner and a pronoun. Interestingly, significant differences were observed only in two-word minimal contexts, with increased posterior temporal activity for nouns between 400 and $600 \mathrm{~ms}$. Importantly, the source-reconstruction analysis was based on a small sample size $(\mathrm{N}=6)$, therefore replication studies including a larger number of participants might be needed to draw stronger conclusions.

Homonyms and homophones. A line of subsequent studies tested noun-verb categorical differences employing homonyms and homophones in minimal syntactic contexts (e.g., "the dance", "they dance"). This manipulation offers two major advantages. First, orthographic and acoustic differences are by definition controlled for, as nouns and verbs share the same form. Notably, this can be achieved not only in English (Burton et al., 2009; Lee \& Federmeier, 2006; Pulvermüller et al., 2012; Tyler et al., 2008), but also in some languages with a more complex morphological system (e.g., Italian, see Tsigka et al., 2014). Second, semantic differences between nouns and verbs can be minimized (Tsigka et al., 2014; Tyler et al., 2008), or included as a factor in the experimental design once the two categories have different meanings (e.g., "the duck" versus "to duck", Lee and Federmeier, 2006).

In a first fMRI study, Tyler et al. (2008) employed homonyms of three types: more frequently used as nouns (e.g., "battle"), more frequently used as verbs (e.g., "drive"), or equally used across the two categories (e.g., "visit"). The homonyms were presented either in isolation or preceded by a determiner or a pronoun. The authors hypothesized that, if differences between the categories depend on the use of 
their grammatical features, noun and verbs would differ at the neural level only in two-word contexts. In line with this hypothesis, verbs elicited stronger activation of the pMTG than nouns, but only when part of a two-word construction. Furthermore, brain response to isolated homonyms was not modulated by the frequency of its use as a noun or a verb. A later fMRI study (Burton et al., 2009) however provided conflicting results, showing increased activation of the posterior temporal lobe for homophones followings determiners (e.g., "the act") than after the particle "to" (e.g., "to act"). Note however that, contrary to the design of Tyler et al. (2008), verbs in these two-word constructions were not part of a full sentence. A role of the posterior temporal lobe in verb processing was however supported by processing two-word constructions with unambiguous verbs (e.g., "to bathe"). Overall, this study provides initial evidence for an interaction between class ambiguity (homophones or unambiguous category) and grammatical class in driving activation of posterior temporal regions, which should be addressed in future studies.

Importantly, a factor which might have been overlooked in these studies is the semantic ambiguity of the homonyms and homophones. Indeed, an ERP study by Lee and Federmeier (2006) showed that two-word constructions including homonyms with ambiguous meaning (e.g., "the duck" and "to duck") elicit a sustained frontal positivity compared to those with an unambiguous one (e.g., "the vote" and "to vote"). This study further showed differences between nouns and verbs in the EEG signal. On the one hand, nouns elicited a stronger N400 than nouns independently from semantic ambiguity. On the other, verbs elicited a larger late positivity (300-700 ms) than nouns, but only for semantically unambiguous conditions. A last fMRI study employing English homonyms (Pulvermüller et al., 2012) showed increased activation of BA44 and the inferior premotor cortex for determiner phrases (e.g., "her walks") compared to sentences (e.g., "she walks"). This study is also particularly important as it shows that activations of the motor system in two-word containing action words is observed in the absence of overt marking (e.g., "she walk", "her walk"), but not for inflected words (e.g., "she walks", "her walks"). This aspect is of great importance for studying the noun-verb distinction, as verbs are more likely to reflect to actions than nouns. Therefore, when contrasting uninflected nouns and verbs, suboptimal stimuli list not matched semantically might drive spurious activation of the motor system for verb processing.

One study, employing Italian homonyms (e.g., "io ballo", I dance, "il ballo", the dance) exploited the high temporal resolution of MEG to highlight differences between determiner phrases and sentences both during the first and second word, across several time-windows (Tsigka et al., 2014). In particular, pronouns elicited stronger activation of the right parietal lobe around $100 \mathrm{~ms}$, and of the anterior frontal regions around $220 \mathrm{~ms}$, compared to determiners. Verbs initially elicited stronger activation of the parietal cortex compared to nouns, and after $300 \mathrm{~ms}$ differences emerged in a left-lateralized fronto-parietal network, comprising the left IFG. Overall, this MEG study did not replicate the increased activation of the posterior temporal lobe for sentences previously observed (Tyler et al., 2008). Given the reduced sample included in the analysis $(\mathrm{N}=12)$ and the use of structural template rather than individual MRI images, it is possible that decreased power and spatial resolution contributed to the diverging findings.

Recent applications and methodological considerations. A more recent MEG study, employing French determiner phrases and sentences with unambiguous nouns and verbs, further observed differences between these two structures already at the first word (Strijkers et al., 2019). In this study, the authors presented nouns and verbs, either following determiners and personal pronouns (e.g., "ta chaise", your chair, "tup prends", you take) or in isolation (i.e., following a series of symbols, matched in length). Compared to pronouns, determiners resulted in increased activation the right pars opercularis of Broca's area, together with a large number of left frontal regions. Differences between nouns and verbs emerged only when they were part of a two-word structure, with increased activation of left and right IFG for the second word of determiner phrases. In the left IFG, this effect was observed with an onset latency of only 80 ms. As the authors pointed out, it is important to ensure that the contrast of nouns and verbs does not merely reflect a prolonged effect stemming from determiner/pronoun differences. Indeed, in this study the signal for nouns and verbs already diverges at the onset of the second word, and a baseline-correction procedure based on the $100 \mathrm{~ms}$ before this point would remove the observed effect. Strijkers et al. (2019) showed that the pre- and post-onset effects were not significantly correlated, therefore ruling out a prolonged nature of the effect in the first word. From a methodological point of view, this study allows to formulate valid design considerations, especially for studies contrasting phrases (e.g., "this table") and lists ("apple table"), where the brain response to the first word might already differ, possibly in a prolonged fashion.

Summary. Several studies point towards the left IFG and PTL as regions differentially representing nouns and verbs, albeit sometimes with effects in opposite directions, once these categories are extracted to build two-word constituents. Further studies are needed, building on the discussed methodological considerations. In this regard, the use of homophones and homonyms, in combination with a careful control of semantic confound, might allow to shed light on the basic distinction between these two grammatical 
categories.

\section{Key aspects of basic syntactic composition}

We have reviewed more than fifty studies, conducted in eleven languages, which have employed basic two-word constructions to investigate the behavioral and neural basis of syntactic processing. Across these studies, four key features of the syntactic combinatorial system have emerged, which are summarized in the following sections (Figure 6).

Combining two words in a constituent is a rule-based process. A first central feature of the syntactic combinatorial system is that it builds structures according to abstract grammatical rules, operating at the categorical and agreement level. Syntactic priming studies (e.g., Goodman et al., 1981; Lukatela et al., 1983, see section 2.1.1) demonstrated that, when the categorical or agreement features of two words are not compatible, linguistic processing is disrupted. Similarly, E/MEG studies showed that categorical and agreement violations elicit syntactic negativities (e.g., Barber and Carreiras, 2003; Hasting and Kotz, 2008; Pulvermüller and Shtyrov, 2003), whose early latency is compatible with a fast and efficient application of grammatical rules. The rule-based nature of syntactic composition is further supported by two sMMN studies (Herrmann et al., 2009, Pulvermüller \& Assadollahi, 2007), comparing grammatical structures of different frequency and ungrammatical ones. In particular, the early stage of syntactic analysis reflects a binary decision: either an utterance follows the rules of a given grammar or it does not, independently from its frequency of occurrence in natural language. This claim is further reinforced by the observation of syntactic priming effects (e.g., Gurjanov, Lukatela, Moskovljević, et al., 1985, Katz et al., 1987; Lukatela et al., 1983; Lukatela et al., 1982) and negativities (Münte \& Heinze, 1994) when grammatical rules are violated in two-word constructions with pseudo-words, which by definition do not occur in natural language. Converging evidence comes from studies which have compared constituents and lists built with pseudo-words (Segaert et al., 2018, Zaccarella \& Friederici, 2015a).

The rule-based combinatorial process strongly relies on the purely syntactic nature of function words (Barber \& Carreiras, 2005, Faussart et al., 1999, Schell et al., 2017) and inflectional morphemes (Lukatela et al., 1983, Lukatela et al., 1982). Indeed, a recent study showed that different function words seem to initiate specific pathways to extract the categorical features of upcoming words (Pyatigorskaya et al., 2021), on which the combinatorial system can operate. Interestingly, when both function words and inflectional particles are missing in a two-word construction (e.g., "red boat"), a different compositional system seems to be engaged, operating on the conceptual features of the two content words (Lukatela, Carello, et al., 1987; Pylkkänen, 2020). This might reflect an early recognition of the impossibility of building a grammatical structure starting

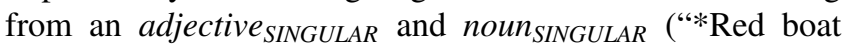
sails down the river"), which would require a function word preceding them ("A red boat sails down the river") or inflectional plural marking present (e.g., "Red boats sail down the river").

Early syntactic analysis occurs automatically, followed by task-dependent processes. Both behavioral and neurophysiological data at the two-word level support an automatic nature of syntactic analysis. In particular, syntactic priming effects have been observed when SOA manipulations reduced the time available for strategic processes (Colé \& Segui, 1994, Katz et al., 1987, Lukatela et al., 1982) and, at least at the categorical level, with subliminal presentation of the prime (Berkovitch \& Dehaene, 2019; Pyatigorskaya et al., 2021). Notably, in this paradigm participants seem unable to avoid analysing the grammatical relationship between prime and target, despite not being necessary to provide a correct answer (Faussart et al., 1999, Seidenberg et al., 1984). Converging evidence for automaticity in syntactic analysis comes from the observation of the ESN during visual distraction conditions (Hasting \& Kotz, 2008) and by several sMMN studies (Hanna et al., 2014; Hasting et al., 2007; Herrmann et al., 2009, Pulvermüller \& Assadollahi, 2007; Pulvermüller et al., 2008; Shtyrov et al., 2003). Both ERP components are characterized by early latency onsets, in line with a fast and efficient application of syntactic analysis.

This first and automatic step of analysis is followed by late controlled processes. At the behavioral level, they might contribute to the larger syntactic priming effects which are observed when the prime is not masked (Berkovitch \& Dehaene, 2019; Pyatigorskaya et al., 2021). At the neural level, a late positivity (P600) follows the early syntactic negativities when participants are performing a task or are not actively distracted (Barber \& Carreiras, 2005; Hasting \& Kotz, 2008, Jakuszeit et al., 2013; Maran et al., 2021). Interestingly, differences between types of agreement violations (i.e. gender vs number) have been described in this time-window (Barber \& Carreiras, 2003, 2005), in line with the notion of longer re-analysis step when the violated syntactic feature (i.e., gender) is expressed by the stem (Faussart et al., 1999).

Overall, the reviewed studies support a two-fold model of syntactic analysis. A first step, occurring in an automatic fashion, provides a binary outcome: either something is grammatical or it is not (Herrmann et al., 2009, Pulvermüller \& Assadollahi, 2007), independently of frequency effects. This diagnostic process is characterised by a fast and efficient analysis of information, as reflected in the early latencies of the ESN (Hasting \& Kotz, 2008) and sMMN (Hasting et al., 2007, Herrmann et al., 2009, Pulvermüller \& 


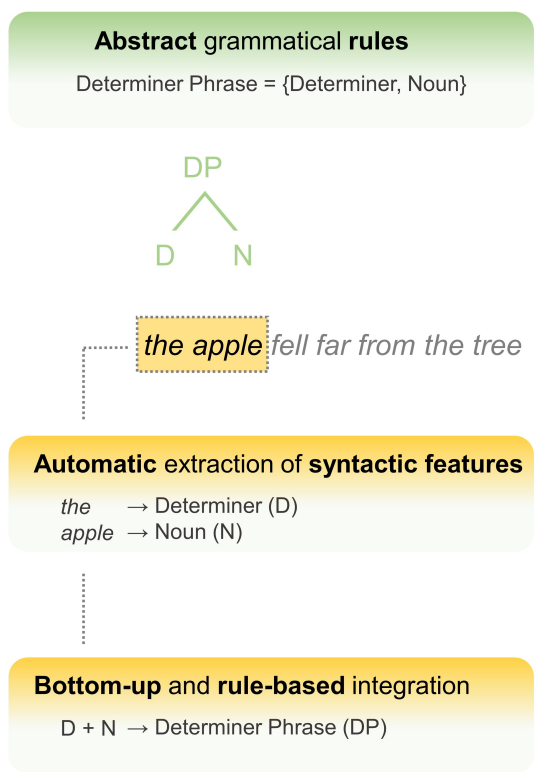

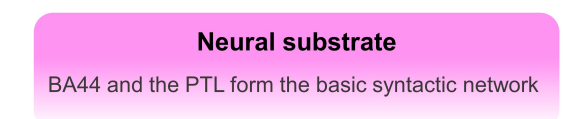

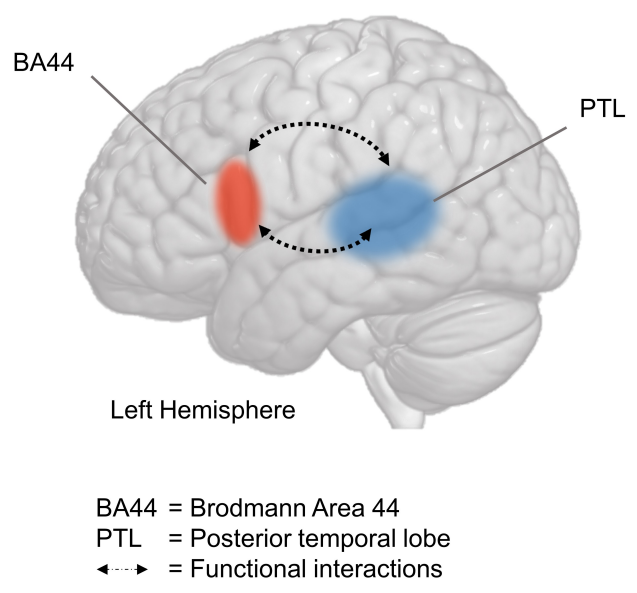

Figure 6

\section{Figure 6}

The tree levels of description of basic syntactic composition. At the formal level, grammar (in green) provides a set of abstract rules which govern the combination of two words into a constituent. At the computational level, grammatical rules are implemented with the automatic extraction and integration of syntactic features (e.g., category) of incoming words. At the neural level, basic syntactic composition relies on a syntactic network composed of BA44 and the PTL, and on the functional interaction between these two regions.

Assadollahi, 2007) components. As a second step, repairing and re-analysis processes can be subsequently engaged in a task-dependent manner, reflected in P600 components. Note that in this time-window fine-grained differences along a continuum might emerge within ungrammatical structures, possibly driven by the depth of the re-analysis and repair processes invoked (Barber \& Carreiras, 2005, Faussart et al., 1999).

The left IFG and posterior temporal lobe are key regions of the syntactic combinatorial system. Data from all the reviewed paradigm (syntactic violations, phrases/sentences versus, comparison of nouns and verbs) support a central role of Broca's area and the posterior temporal lobe in analysing and combining grammatical information in two-word constituents. Several fMRI and MEG studies showed an involvement of Broca's area in detecting an incompatibility between the syntactic features of an incoming word and the preceding one, both at the categorical level (Herrmann et al., 2012) and agreement level (Carreiras et al., 2010; Hanna et al., 2014; Heim et al., 2010). Additional activations vary according to the type of violation, as categorical errors engage the bilateral STS (Herrmann et al., 2012), while the premotor cortex and the parietal lobe are activated by agreement errors (Carreiras et al., 2010, Heim et al., 2010). Even in the absence of violations, an involvement of the left IFG (Schell et al., 2017, Wu et al., 2019, Zaccarella \& Friederici, 2015a) and posterior temporal regions (Matar et al., 2021; Schell et al., 2017, Wu et al., 2019) in syntactic composition is supported by the phrase-versus-list comparisons, with a stronger activations of these regions driven by hierarchical syntactic processing. Importantly, the involvement of these two regions in syntactic composition has been observed both in the auditory (Heim et al., 2010; Herrmann et al., 2012, Schell et al., 2017) and visual (Carreiras et al., 2010; Heim et al., 2010; Matar et al., 2021; Wu et al., 2019, Zaccarella \& Friederici, 2015a) modality, pointing towards modality-independent operations in these regions. This is further supported by recent studies showing that the left IFG (Trettenbrein et al., 2020) and the posterior temporal lobe (Matchin et al., 2022) are central regions in processing signed languages, in line with the abstract nature of their linguistic operations. Similarly, recent 
evidence from artificial grammar paradigms supports the involvement of the left IFG and PTL in abstract categorical processes (Chen et al., 2021, Chen et al., 2019), putting forward the working hypothesis that dissociations between left IFG and pTL in syntactic composition can be traced-the left IFG being specialized in building up hierarchies on the basis of categorical features, the PTL integrating hierarchies with other sources of linguistic information, including meaning. Under this account, the information exchange between the left IFG and PTL would allow to reconstruct the hierarchical dependencies characterizing human language, providing an analysis that will also interface with the semantic system.

Interestingly, the same regions activated by the detection of syntactic violations and hierarchical processing (i.e., left IFG and pMTG/STG) seem to be involved in processing differences between constituents formed with nouns and verbs (Burton et al., 2009; Pulvermüller et al., 2012; Strijkers et al., 2019, Tsigka et al., 2014; Tyler et al., 2008). An interesting hypothesis is that the effects observed in the left fronto-temporal syntactic network reflect the different load on the combinatorial mechanism when building constituents with nouns or verbs, rather than the categorical effects per se. While, at least in languages with a rich inflectional system, both nouns and verbs might engage to a similar extent morphological analysis, only the latter category requires the analysis of tense features (which can be represented as an additional node, Tense Phrase, in the derivation) and assigns thematic roles. Indeed, two studies have observed categorical effects in the left IFG (Strijkers et al., 2019) or the pMTG (Tyler et al., 2008) when two-word constructions are employed, but not for nouns, verbs or homonyms presented in isolation. Further studies are needed to shed light on this hypothesis, in line with the theoretical (Vigliocco et al., 2011) and methodological (Crepaldi et al., 2013; Crepaldi et al., $2011)$ considerations previously pointed out.

Overall, the reviewed studies support the notion of a neuro-anatomical dissociation between the syntactic and semantic combinatorial processes when building basic two-word structures. In particular, the posterior portion of the left IFG (BA44) and temporal lobe combine abstract syntactic information into constituents, while the ATL, vmPFC (Pylkkänen, 2020), angular gyrus and more anterior portion of the left IFG (Graessner, Zaccarella, Friederici, et al., 2021, Graessner, Zaccarella, \& Hartwigsen, 2021) are involved in semantic and conceptual composition. Initial evidence exists also for a modality-independent nature of this dissociation (Blanco-Elorrieta et al., 2018, Matchin et al., 2022, Moreno et al., 2018), which should be addressed in future studies.

Syntactic composition as an efficient bottom-up process. In recent years interest has grown in understanding the contribution of top-down prediction and bottom-up integration in incremental syntactic composition (e.g., left-corner, bottomup and top-down parsing, see Abney and Johnson, 1991; Resnik, 1992). In particular, several studies have correlated metrics based on computational linguistics parsers with neural signals recorded while participants listen to narratives, looking for a convergence between parsing operations and brain functioning (Bhattasali et al., 2019; Brennan \& Hale, 2019; Brennan \& Martin, 2020; Brennan \& Pylkkänen, 2017, Brennan et al., 2016, Hale et al., 2018). A limitation of this approach is that syntactic and semantic composition become difficult to disentangle, as each incoming word of the narratives builds not only grammatical information but also semantic one (Bhattasali et al., 2019). Two-word constructions might give complementary insights, as they allow to isolate predictive and integration processes, occurring respectively during the first and second word of the utterance.

The reviewed studies provide initial evidence for fast and efficient bottom-up operations in syntactic composition. At the behavioral level, syntactic priming effects have been related to a post-lexical stage of processing (Carello et al., 1988. Seidenberg et al., 1984), rather than pre-activation of lexical entries, which would be inefficient as a very large cohort of candidates (e.g., all the nouns and adjectives) would be activated following a function word (e.g., a determiner). This suggests the existence of operations efficiently integrating the syntactic features of incoming words into the preceding context, in line with the notion of bottom-up parsing (Hale, 2014). Converging evidence comes from the inhibitory nature of syntactic priming (Friederici, Steinhauer, et al., 1999, Pyatigorskaya et al., 2021). If top-down predictions were at work, faster RTs should be observed for a grammatical two-word phrase compared to a matched baseline. This however is not the case, and syntactic priming rather stems from longer RTs of the ungrammatical condition compared to a neutral baseline (Friederici, Steinhauer, et al., 1999; Pyatigorskaya et al., 2021). In this regard, some sMMN studies have shown an opposite pattern, with the response to a grammatical construction differing words presented (Pulvermüller \& Shtyrov, 2003, 2006), possibly reflecting facilitation process. A possible explanation is that, since only two constructions are used in sMMN paradigms, function words could be used to predict a specific lexical item, therefore facilitation can be observed. In paradigms which use a larger number of lexical items (e.g., syntactic priming), that might not be the case, and only reliable inhibition for ungrammatical structures is observed. As discussed above and previously in the literature (Seidenberg et al., 1984), the syntactic features of a given word are compatible with a large number of candidates in the lexicon that would be inefficient to pre-activate. Indeed, even if many candidates (e.g., all the nouns and adjectives) were pre-activated, mechanisms such lateral inhibition and lexical competition would remove any facilitatory effect. This might constitute a 
key difference with the semantic system, for which top-down prediction might be more efficient, given that few specific lexical items can be pre-activated, therefore giving rise to facilitation compared to neutral baselines (Lukatela, Carello, et al., 1987).

Neuro-imaging studies employing syntactic violations in which grammaticality is orthogonal to the identity of the first word and the syntactic features of the second one (Carreiras et al., 2010, Hasting \& Kotz, 2008; Heim et al., 2010, Herrmann et al., 2012; Maran et al., 2021) allow to highlight the neural correlates of bottom-up integration, as by definition any virtual predictive processes is subtracted out These studies have shown the presence of syntactic negativities (Barber \& Carreiras, 2005; Hasting \& Kotz, 2008, Maran et al., 2021), suggesting the fast analysis of two words' syntactic feature compatibility. In parallel, they have provided evidence for a key role of Broca's area in this process, both with respect to agreement (Carreiras et al., 2010, Heim et al., 2010) and categorical (Herrmann et al., 2012) features. Converging evidence comes also from two TMS studies, showing that disruption of Broca's area during the integration stage (i.e., the second word of a two-word construction) affects syntactic processing (Carreiras et al., 2012), while stimulation of this region at the predictive phase does not interfere with this process (Maran et al., 2021). Finally, a role of Broca's area in bottom-up syntactic composition is also supported by a phrase-versus-list study, which included a one-word condition controlling for potential predictive effects (Zaccarella \& Friederici, 2015a).

Overall, the reviewed studies support a crucial role of bottom-up process in integrating the syntactic features of two words into a constituent. This reliance on basic bottomup operations, with a limited role of top-down predictions, might represent a critical distinction between the syntactic combinatorial system and the semantic one, possibly grounded in differences at the neuro-anatomical level (Graessner, Zaccarella, Friederici, et al., 2021; Graessner, Zaccarella, \& Hartwigsen, 2021; Pylkkänen, 2020, Zaccarella \& Friederici, 2015a, Zaccarella, Schell, et al., 2017). Similarly, this aspect might differentiate syntactic composition, characterised by hierarchical processing, from nonlinguistic domains which might strongly rely on serial topdown predictions (Zaccarella et al., 2021).

\section{Conclusions and future directions}

Two-word studies help characterizing key aspects of the neuro-cognitive basis of syntactic composition. The reviewed studies allowed also to formulate important methodological considerations. The use of orthogonal designs, cross-splicing techniques and difference waves for ERP studies might allow addressing important methodological issues, previously pointed out in syntactic studies (Steinhauer \& Drury, 2012). While at present syntactic composition has not been tested in signed languages, (but see Blanco-Elorrieta et al., 2018, for an application of the "red boat" paradigm), recent methodological advances and norms development allow to test this process directly, while achieving great control over perceptual and psycholinguistic variables of interest (Bungeroth et al., 2008, Caselli et al., 2017, Sehyr et al., 2021; Trettenbrein et al., 2021; Trettenbrein \& Zaccarella, 2021).

Based on the evidence reviewed in this article, methodological advances now make it possible to progress towards a mechanistic understanding of syntactic composition at the neural and cognitive levels (Figure 6). Fundamental research questions include how the information flows within the syntactic network, how labels are applied to recursively build hierarchical structures, and which are the key phylogenetic and ontogenetic changes which made the human brain an efficient integrator of syntactic features. We thus believe that a coherent research program that integrates hypotheses from linguistic theory can help reveal how exactly composition occurs at this fundamental level, thus providing the basis for understanding the uniquely human ability to use language.

\section{Acknowledgements}

The authors would like to thank Patrick Trettenbrein and Elena Pyatigorskaya for the valuable discussions on the present work.

\section{References}

Abney, S. P., \& Johnson, M. (1991). Memory requirements and local ambiguities of parsing strategies. Journal of Psycholinguistic Research, 20(3), 233-250.

Ansorge, U., Reynvoet, B., Hendler, J., Oettl, L., \& Evert, S. (2013). Conditional automaticity in subliminal morphosyntactic priming. Psychological Research, 77(4), 399-421. https://doi.org/10.1007/s00426012-0442-z

Arcara, G., Franzon, F., Gastaldon, S., Brotto, S., Semenza, C., Peressotti, F., \& Zanini, C. (2019). One can be some but some cannot be one: ERP correlates of numerosity incongruence are different for singular and plural. Cortex, 116, 104-121. https://doi.org/ 10.1016/j.cortex.2018.10.022

Arcara, G., Pezzetta, R., Varela, S. B., Rizzi, G., Formica, S., Turco, C., Piccione, F., \& Semenza, C. (2021). Magnetoencephalography reveals differences in brain activations for fast and slow responses to simple multiplications. Scientific Reports, 1-13. https: //doi.org/10.1038/s41598-021-97927-8 
Baillet, S. (2017). Magnetoencephalography for brain electrophysiology and imaging. Nature Neuroscience, 20, 327-339. https://doi.org/https://doi.org/10. $1038 / \mathrm{nn} .4504$

Barber, H., \& Carreiras, M. (2003). Integrating gender and number information in Spanish word pairs: an ERP study. Cortex, 39(3), 465-482. https://doi.org/https: //doi.org/10.1016/S0010-9452(08)70259-4

Barber, H., \& Carreiras, M. (2005). Grammatical gender and number agreement in Spanish: An ERP comparison. Journal of Cognitive Neuroscience, 17(1), 137-153. https://doi.org/10.1162/0898929052880101

Bates, E., Devescovi, A., Hernandez, A., \& Pizzamiglio, L. (1996). Gender priming in Italian. Perception Psychophysics, 58(7), 992-1004.

Bemis, D. K., \& Pylkkänen, L. (2011). Simple composition: a magnetoencephalography investigation into the comprehension of minimal linguistic phrases. The Journal of neuroscience, 31(8), 2801-2814. https : //doi.org/10.1523/JNEUROSCI.5003-10.2011

Benítez-Burraco, A., \& Murphy, E. (2019). Why Brain Oscillations Are Improving Our Understanding of Language. Frontiers in Behavioral Neuroscience, 13(August), 1-10. https://doi.org/10.3389/fnbeh. 2019.00190

Berkovitch, L., \& Dehaene, S. (2019). Subliminal syntactic priming. Cognitive Psychology, 109(December 2018), 26-46. https://doi.org/10.1016/j.cogpsych. 2018.12.001

Berwick, R. C., Friederici, A. D., Chomsky, N., \& Bolhuis, J. J. (2013). Evolution, brain, and the nature of language. Trends in Cognitive Sciences, 17(2), 98. https://doi.org/10.1016/j.tics.2012.12.002

Bhattasali, S., Fabre, M., Luh, W. M., Al Saied, H., Constant, M., Pallier, C., Brennan, J. R., Spreng, R. N., \& Hale, J. (2019). Localising memory retrieval and syntactic composition: an fMRI study of naturalistic language comprehension. Language, Cognition and Neuroscience, 34(4), 491-510. https://doi.org/10. 1080/23273798.2018.1518533

Blanco-Elorrieta, E., Kastner, I., Emmorey, K., \& Pylkkänen, L. (2018). Shared neural correlates for building phrases in signed and spoken language. Scientific Reports, 8(1), 1-10. https://doi.org/10.1038/ s41598-018-23915-0

Bock, J. K. (1986). Syntactic Persistence in Language. Cognitive Psychology, 18, 355.387. https:// doi.org/ https://doi.org/10.1016/0010-0285(86)90004-6

Bozic, M., Fonteneau, E., Su, L., \& Marslen-Wilson, W. D. (2015). Grammatical analysis as a distributed neurobiological function. Human Brain Mapping, 36(3), 1190-1201. https://doi.org/10.1002/hbm. 22696
Brennan, J. R., \& Hale, J. T. (2019). Hierarchical structure guides rapid linguistic predictions during naturalistic listening. Plos One, 14(1), e0207741. https://doi. org/10.1371/journal.pone.0207741

Brennan, J. R., \& Martin, A. E. (2020). Phase synchronization varies systematically with linguistic structure composition. Philosophical Transactions of the Royal Society B: Biological Sciences, 375(1791), 20190305. https://doi.org/10.1098/rstb.2019.0305

Brennan, J. R., \& Pylkkänen, L. (2017). MEG Evidence for Incremental Sentence Composition in the Anterior Temporal Lobe. Cognitive Science, 41, 1515-1531. https://doi.org/10.1111/cogs.12445

Brennan, J. R., Stabler, E. P., Van Wagenen, S. E., Luh, W. M., \& Hale, J. T. (2016). Abstract linguistic structure correlates with temporal activity during naturalistic comprehension. Brain and Language, 157-158, 81-94. https://doi.org/10.1016/j.bandl. 2016.04.008

Brunellière, A., Franck, J., Ludwig, C., \& Frauenfelder, U. H. (2007). Early and automatic syntactic processing of person agreement. NeuroReport, 18(6), 537-541. https://doi.org/10.1097/WNR.0b013e3280b07ba1

Bungeroth, J., Stein, D., Dreuw, P., Ney, H., Morrissey, S., Way, A., \& Van Zijl, L. (2008). The ATIS sign language corpus. Proceedings of the 6th International Conference on Language Resources and Evaluation, LREC 2008, 2943-2946.

Burton, M. W., Krebs-Noble, D., Gullapalli, R., \& Berndt, R. S. (2009). Functional neuroimaging of grammatical class: Ambiguous and unambiguous nouns and verbs. Cognitive Neuropsychology, 26(2), 148-171. https://doi.org/10.1080/02643290802536090

Buzsáki, G., \& Draguhn, A. (2004). Neuronal Oscillations in Cortical Networks. Science, 304(June), 1926-1930. https://doi.org/https://doi.org/10.1126/science. 1099745

Carello, C., Lukatela, G., \& Turvey, M. T. (1988). Rapid naming is affected by association but not by syntax. Memory Cognition, 16(3), 187-195. https://doi. org/10.3758/BF03197751

Carreiras, M., Carr, L., Barber, H. A., \& Hernandez, A. (2010). Where syntax meets math: Right intraparietal sulcus activation in response to grammatical number agreement violations. NeuroImage, 49(2), 1741-1749. https://doi.org/10.1016/j.neuroimage. 2009.09 .058

Carreiras, M., Pattamadilok, C., Meseguer, E., Barber, H., \& Devlin, J. T. (2012). Broca's area plays a causal role in morphosyntactic processing. Neuropsychologia, 50(5), 816-820. https : / / doi . org / 10 . 1016/j . neuropsychologia.2012.01.016 
Caselli, N. K., Sehyr, Z. S., Cohen-Goldberg, A. M., \& Emmorey, K. (2017). ASL-LEX: A lexical database of American Sign Language. Behavior Research Methods, 49(2), 784-801. https://doi.org/10.3758/ s13428-016-0742-0

Chen, L., Goucha, T., Männel, C., Friederici, A. D., \& Zaccarella, E. (2021). Hierarchical syntactic processing is beyond mere associating: Functional magnetic resonance imaging evidence from a novel artificial grammar. Human Brain Mapping, (March), hbm.25432. https://doi.org/10.1002/hbm.25432

Chen, L., Wu, J., Fu, Y., Kang, H., \& Feng, L. (2019). Neural substrates of word category information as the basis of syntactic processing. Human Brain Mapping, 40(2), 451-464. https://doi.org/10.1002/hbm.24386

Chomsky, N. (1995). The minimalist program. MIT press.

Colé, P., \& Segui, J. (1994). Grammatical incongruency and vocabulary types. Memory cognition, 22(4), 387394. https :// doi .org/https:// doi . org/10.3758/ BF03200865

Crepaldi, D., Berlingeri, M., Cattinelli, I., Borghese, N. A., \& Luzzatti, C. (2013). Clustering the lexicon in the brain: a meta-analysis of the neurofunctional evidence on noun and verb processing. Frontiers in Human Neuroscience, 7(June), 1-15. https://doi . org/10.3389/fnhum.2013.00303

Crepaldi, D., Berlingeri, M., Paulesu, E., \& Luzzatti, C. (2011). A place for nouns and a place for verbs? A critical review of neurocognitive data on grammatical-class effects. Brain and Language, 116(1), 33-49. https://doi.org/10.1016/j.bandl . 2010.09.005

Dehaene, S., Piazza, M., Pinel, P., \& Cohen, L. (2003). Three parietal circuits for number processing. Cognitive Neuropsychology, 20(3-6), 487-506. https:// doi . org/10.1080/02643290244000239

Everaert, M. B., Huybregts, M. A., Chomsky, N., Berwick, R. C., \& Bolhuis, J. J. (2015). Structures, Not Strings: Linguistics as Part of the Cognitive Sciences. Trends in Cognitive Sciences, 19(12), 729743. https://doi.org/10.1016/j.tics.2015.09.008

Faussart, C., Jakubowicz, C., \& Costes, M. (1999). Gender and number processing in spoken French and Spanish. The Italian Journal of Linguistics, 11(1), 75102.

Fedorenko, E., Blank, I. A., Siegelman, M., \& Mineroff, Z. (2020). Lack of selectivity for syntax relative to word meanings throughout the language network. Cognition, 203(November 2018). https://doi .org/ 10.1016/j.cognition.2020.104348

Fedorenko, E., Scott, T. L., Brunner, P., Coon, W. G., Pritchett, B., Schalk, G., \& Kanwisher, N. (2016). Neural correlate of the construction of sentence meaning.
Proceedings of the National Academy of Sciences of the United States of America, 113(41), E6256E6262. https://doi.org/10.1073/pnas.1612132113

Fiebach, C. J., Maess, B., \& Friederici, A. D. (2002). Neuromagnetic evidence that differences in noun and verb processing are modulated by the presence of a syntactic context. Proceedings of the 13th International Conference on Biomagnetism, 5, 339-341.

Fló, E., Cabana, Á., \& Valle-Lisboa, J. C. (2020). EEG signatures of elementary composition: Disentangling genuine composition and expectancy processes. Brain and Language, 209(July), 104837. https://doi.org/10.1016/j.bandl.2020.104837

Forster, K. I. (1981). Priming and the effects of sentence and lexical contexts on naming time: Evidence for autonomous lexical processing. The Quarterly Journal of Experimental Psychology Section A, 33(4), 465-495. https : / / doi . org / 10 . 1080/ 14640748108400804

Friederici, A. D. (2011). The brain basis of language processing: From structure to function. Physiological Reviews, 91(4), 1357-1392. https://doi.org/10.1152/ physrev.00006.2011

Friederici, A. D., Chomsky, N., Berwick, R. C., Moro, A., \& Bolhuis, J. J. (2017). Language, mind and brain. Nature Human Behaviour, 1(10). https://doi.org/10. 1038/s41562-017-0184-4

Friederici, A. D., \& Jacobsen, T. (1999). Processing grammatical gender during language comprehension. Journal of Psycholinguistic Research, 28(5), 467484. https://doi.org/10.1023/A:1023264209610

Friederici, A. D., Pfeifer, E., \& Hahne, A. (1993). Eventrelated brain potentials during natural speech processing: effects of semantic, morphological and syntactic violations. Cognitive Brain Research, l(3), 183-192. https://doi . org/10 . 1016/09266410(93)90026-2

Friederici, A. D., \& Singer, W. (2015). Grounding language processing on basic neurophysiological principles. Trends in Cognitive Sciences, 19(6), 329338. https://doi.org/10.1016/j.tics.2015.03.012 Very interesting

Friederici, A. D., Steinhauer, K., \& Frisch, S. (1999). Lexical integration: Sequential effects of syntactic and semantic information. Memory and Cognition, 27(3), 438-453. https://doi.org/10.3758/BF03211539

Friederici, A. D., Von Cramon, D. Y., \& Kotz, S. A. (1999). Language related brain potentials in patients with cortical and subcortical left hemisphere lesions. Brain, 122(6), 1033-1047. https://doi.org/10.1093/ brain/122.6.1033

Fries, P. (2015). Perspective Rhythms for Cognition : Communication through Coherence. Neuron, 88(1), 
220-235. https://doi.org/10.1016/j.neuron.2015.09. 034

Goodman, G., McClelland, J., \& Gibbs Jr., R. (1981). The role of syntactic context in word recognition. https: //doi.org/10.3758/BF03202352

Goucha, T., \& Friederici, A. D. (2015). The language skeleton after dissecting meaning: A functional segregation within Broca's Area. NeuroImage, 114, 294302. https://doi.org/10.1016/j.neuroimage.2015.04. 011

Graessner, A., Zaccarella, E., Friederici, A. D., Obrig, H., \& Hartwigsen, G. (2021). Dissociable contributions of frontal and temporal brain regions to basic semantic composition. Brain Communications, 3(2). https://doi.org/10.1093/braincomms/fcab090

Graessner, A., Zaccarella, E., \& Hartwigsen, G. (2021). Differential contributions of left-hemispheric language regions to basic semantic composition. Brain Structure and Function, (0123456789). https://doi.org/ 10.1007/s00429-020-02196-2

Gurjanov, M., Lukatela, G., Lukatela, K., Savić, M., \& Turvey, M. T. (1985). Grammatical Priming of Inflected Nouns by the Gender of Possessive Adjectives. Journal of Experimental Psychology: Learning, Memory, and Cognition, 11(4), 692-701. https: //doi.org/10.1037/0278-7393.11.1-4.692

Gurjanov, M., Lukatela, G., Moskovljević, J., Savić, M., \& Turvey, M. T. (1985). Grammatical priming of inflected nouns by inflected adjectives. Cognition, 19(1), 55-71. https : // doi . org / 10 . 1016/0010 0277(85)90031-9

Hagoort, P. (2015). MUC (Memory, Unification, Control): A Model on the Neurobiology of Language Beyond Single Word Processing. Elsevier Inc. https://doi. org/10.1016/B978-0-12-407794-2.00028-6

Hagoort, P., \& Indefrey, P. (2014). The neurobiology of language beyond single words. Annual Review of Neuroscience, 37, 347-362. https://doi.org/10.1146/ annurev-neuro-071013-013847

Hahne, A., \& Friederici, A. D. (1999). Electrophysiological evidence for two steps in syntactic analysis. Early automatic and late controlled processes. Journal of Cognitive Neuroscience, 11(2), 194-205.

Hale, J. T. (2014). Automaton theories of human sentence comprehension. CSLI Publications.

Hale, J. T., Dyer, C., Kuncoro, A., \& Brennan, J. R. (2018). Finding Syntax in Human Encephalography with Beam Search. (2014). https://doi.org/10.1023/A: 1019527211183

Hanna, J., Mejias, S., Schelstraete, M. A., Pulvermüller, F., Shtyrov, Y., \& van der Lely, H. K. (2014). Early activation of Broca's area in grammar processing as revealed by the syntactic mismatch negativity and distributed source analysis. Cognitive Neuroscience, 5(2), 66-76. https :// doi .org/10 . 1080/ 17588928.2013 .860087

Hanna, J., Shtyrov, Y., Williams, J., \& Pulvermüller, F. (2016). Early neurophysiological indices of second language morphosyntax learning. Neuropsychologia, 82, 18-30. https : / / doi . org / 10 . 1016/j . neuropsychologia.2016.01.001

Hartwigsen, G. (2015). The neurophysiology of language: Insights from non-invasive brain stimulation in the healthy human brain. Brain and Language, 148, 8194. https://doi.org/10.1016/j.bandl.2014.10.007

Hasting, A. S., \& Kotz, S. A. (2008). Speeding up syntax: On the relative timing and automaticity of local phrase structure and morphosyntactic processing as reflected in event-related brain potentials. Journal of Cognitive Neuroscience, 20(7), 12071219. https://doi.org/10.1162/jocn.2008.20083

Hasting, A. S., Kotz, S. A., \& Friederici, A. D. (2007). Setting the stage for automatic syntax processing: The mismatch negativity as an indicator of syntactic priming. Journal of Cognitive Neuroscience, 19(3), 386-400. https://doi.org/10.1162/jocn.2007.19.3. 386

Heim, S., Van Ermingen, M., Huber, W., \& Amunts, K. (2010). Left cytoarchitectonic BA 44 processes syntactic gender violations in determiner phrases. $\mathrm{Hu}$ man Brain Mapping, 31(10), 1532-1541. https :// doi.org/10.1002/hbm.20957

Herrmann, B., Maess, B., Hasting, A. S., \& Friederici, A. D. (2009). Localization of the syntactic mismatch negativity in the temporal cortex: An MEG study. NeuroImage, 48(3), 590-600. https://doi.org/10.1016/j. neuroimage.2009.06.082

Herrmann, B., Obleser, J., Kalberlah, C., Haynes, J. D., \& Friederici, A. D. (2012). Dissociable neural imprints of perception and grammar in auditory functional imaging. Human Brain Mapping, 33(3), 584595. https://doi.org/10.1002/hbm.21235

Hultén, A., Schoffelen, J. M., Uddén, J., Lam, N. H., \& Hagoort, P. (2019). How the brain makes sense beyond the processing of single words - An MEG study. NeuroImage, 186, 586-594. https:// doi.org/10. 1016/j.neuroimage.2018.11.035

Iwabuchi, T., Nakajima, Y., \& Makuuchi, M. (2019). Neural architecture of human language: Hierarchical structure building is independent from working memory. Neuropsychologia, 132, 107137. https://doi.org/10. 1016/j.neuropsychologia.2019.107137

Jakuszeit, M., Kotz, S. A., \& Hasting, A. S. (2013). Generating predictions: Lesion evidence on the role of left inferior frontal cortex in rapid syntactic analy- 
sis. Cortex, 49(10), 2861-2874. https://doi.org/10. 1016/j.cortex.2013.05.014

Kang, A. M., Constable, R. T., Gore, J. C., \& Avrutin, S. (1999). An Event-Related fMRI Study of Implicit Phrase-Level Syntactic and Semantic Processing. NeuroImage, 10, 555-561.

Katz, L., Boyce, S., Goldstein, L., \& Lukatela, G. (1987). Grammatical information effects in auditory word recognition. Cognition, 25(3), 235-263. https://doi. org/10.1016/S0010-0277(87)80005-7

Kochari, A., Lewis, A., Schoffelen, J.-M., \& Schriefers, H. (2021). Semantic and syntactic composition of minimal adjective-noun phrases in Dutch: an MEG study. Neuropsychologia, 107754. https://doi.org/ 10.1016/j.neuropsychologia.2021.107754

Lam, N. H., Schoffelen, J. M., Uddén, J., Hultén, A., \& Hagoort, P. (2016). Neural activity during sentence processing as reflected in theta, alpha, beta, and gamma oscillations. NeuroImage, 142, 43-54. https://doi.org/10.1016/j.neuroimage.2016.03.007

Lee, C.-L., \& Federmeier, K. D. (2006). To mind the mind: An event-related potential study of word class and semantic ambiguity. Brain Research, 1081(1), 191202. https://doi.org/10.1016/j.brainres.2006.01.058

Lewis, A. G., Wang, L., \& Bastiaansen, M. (2015). Fast oscillatory dynamics during language comprehension: Unification versus maintenance and prediction? Brain and Language, 148, 51-63. https://doi. org/10.1016/j.bandl.2015.01.003

Lucchese, G., Hanna, J., Autenrieb, A., Miller, T. M., \& Pulvermüller, F. (2017). Electrophysiological Evidence for Early and Interactive Symbol Access and Rule Processing in Retrieving and Combining Language Constructions. Journal of Cognitive Neuroscience, 29(2), 254-266. https://doi.org/10.1162/jocn_a_ 01038

Lucchese, G., Pulvermüller, F., Stahl, B., Dreyer, F. R., \& Mohr, B. (2017). Therapy-Induced Neuroplasticity of Language in Chronic Post Stroke Aphasia: A Mismatch Negativity Study of (A)Grammatical and Meaningful/less Mini-Constructions. Frontiers in human neuroscience, 10, 1-14. https://doi.org/ https://doi.org/10.3389/fnhum.2016.00669

Luck, S. J. (2014). An introduction to the event-related potential technique. MIT press.

Lukatela, G., Carello, C., Kostic, A., \& Turvey, M. T. (1987). Low constraint facilitation in lexical decision with single word contexts. American Journal of Psychology, 90, 93-104.

Lukatela, G., Kostić, A., Feldman, L. B., \& Turvey, M. T. (1983). Grammatical priming of inflected nouns. Memory Cognition, 11(1), 59-63. https://doi.org/ 10.3758/BF03197662
Lukatela, G., Kostić, A., Todorović, D., Carello, C., \& Turvey, M. T. (1987). Type and number of violations and the grammatical congruency effect in lexical decision. Psychological Research, 49(1), 37-43. https://doi.org/10.1007/BF00309201

Lukatela, G., Moraca, J., Stojnov, D., Savic, M. D., Katz, L., \& Turvey, M. T. (1982). Grammatical priming effects between pronouns and inflected verb forms. Psychological Research, 44(4), 297-311. https:// doi.org/10.1007/BF00309326

Maess, B., Schröger, E., \& Widmann, A. (2016). High-pass filters and baseline correction in M/EEG analysis. Commentary on: "How inappropriate high-pass filters can produce artefacts and incorrect conclusions in ERP studies of language and cognition". Journal of Neuroscience Methods, 266, 164-165. https: //doi.org/10.1016/j.jneumeth.2015.12.003

Makuuchi, M., Bahlmann, J., Anwander, A., \& Friederici, A. D. (2009). Segregating the core computational faculty of human language from working memory. Proceedings of the National Academy of Sciences of the United States of America, 106(20), 8362-8367. https://doi.org/10.1073/pnas.0810928106

Maran, M., Numssen, O., Hartwigsen, G., Friederici, A. D., \& Zaccarella, E. (2021). Towards a causal role of Broca's area in language: A TMS-EEG study on syntactic prediction. bioRxiv, 2021.04.14.439631. https://doi.org/10.1101/2021.04.14.439631

Marslen-Wilson, W. D., \& Tyler, L. K. (1980). The temporal structure of spoken language understanding. Cognition, 8(1), 1-71. https://doi.org/http://dx.doi.org/ 10.1016/0010-0277(80)90015-3

Martin, A. E., \& Baggio, G. (2020). Modelling meaning composition from formalism to mechanism. Philosophical Transactions of the Royal Society B: Biological Sciences, 375(1791), 1-7. https://doi.org/ 10.1098/rstb.2019.0298

Matar, S., Dirani, J., Marantz, A., \& Pylkkänen, L. (2021). Left posterior temporal cortex is sensitive to syntax within conceptually matched Arabic expressions. Scientific Reports, 11(1), 7181. https://doi.org/10. 1038/s41598-021-86474-x

Matchin, W., Hammerly, C., \& Lau, E. (2017). The role of the IFG and pSTS in syntactic prediction: Evidence from a parametric study of hierarchical structure in fMRI. Cortex, 88, 106-123. https://doi.org/10. 1016/j.cortex.2016.12.010

Matchin, W., \& Hickok, G. (2020). The Cortical Organization of Syntax. Cerebral Cortex, 30(3), 1481-1498. https://doi.org/10.1093/cercor/bhz180

Matchin, W., İlkbaşaran, D., Hatrak, M., Roth, A., Villwock, A., Halgren, E., \& Mayberry, R. I. (2022). The Cortical Organization of Syntactic Processing 
Is Supramodal: Evidence from American Sign Language. Journal of Cognitive Neuroscience, 34(2), 224-235. https://doi.org/10.1162/jocn_a_01790

Meyer, L. (2018). The neural oscillations of speech processing and language comprehension: state of the art and emerging mechanisms. European Journal of Neuroscience, 48(7), 2609-2621. https://doi.org/ 10.1111/ejn.13748

Molinaro, N., Barber, H. A., Caffarra, S., \& Carreiras, M. (2015). On the left anterior negativity (LAN): The case of morphosyntactic agreement: A Reply to Tanner etal. Cortex, 66, 156-159. https://doi.org/ 10.1016/j.cortex.2014.06.009

Moreno, A., Limousin, F., Dehaene, S., \& Pallier, C. (2018). Brain correlates of constituent structure in sign language comprehension. NeuroImage, 167, 151-161. https://doi.org/10.1016/j.neuroimage.2017.11.040

Münte, T. F., \& Heinze, H. J. (1994). ERP negativities during syntactic processing of written words. In H. J. Heinze, T. F. Münte, \& G. R. Mangun (Eds.), Cognitive electrophysiology. Birkhäuser. https :// doi . org/https://doi.org/10.1007/978-1-4612-0283-7_9

Münte, T. F., Heinze, H. J., \& Mangun, G. R. (1993). Dissociation of brain activity related to syntactic and semantic aspects of language. Journal of cognitive neuroscience, 5(3), 335-44. https://doi . org/10. 1162/jocn.1993.5.3.335

Murphy, E. (2015). The brain dynamics of linguistic computation. Frontiers in Psychology, 6(OCT), 1-19. https://doi.org/10.3389/fpsyg.2015.01515

Näätänen, R., Gaillard, A. W., \& Mäntysalo, S. (1978). Early selective-attention effect on evoked potential reinterpreted. Acta Psychologica, 42(4), 313-329. https://doi.org/10.1016/0001-6918(78)90006-9

Näätänen, R., Paavilainen, P., Rinne, T., \& Alho, K. (2007). The mismatch negativity (MMN) in basic research of central auditory processing: A review. Clinical Neurophysiology, 118(12), 2544-2590. https://doi. org/10.1016/j.clinph.2007.04.026

Näätänen, R., Pakarinen, S., Rinne, T., \& Takegata, R. (2004). The mismatch negativity (MMN): Towards the optimal paradigm. Clinical Neurophysiology, 115(1), 140-144. https://doi.org/10.1016/j.clinph. 2003.04.001

Nelson, M. J., El Karoui, I., Giber, K., Yang, X., Cohen, L., Koopman, H., Cash, S. S., Naccache, L., Hale, J. T., Pallier, C., \& Dehaene, S. (2017). Neurophysiological dynamics of phrase-structure building during sentence processing. Proceedings of the National Academy of Sciences of the United States of America, 114(18), E3669-E3678. https:// doi .org/10. 1073/pnas.1701590114
Neufeld, C., Kramer, S. E., Lapinskaya, N., Heffner, C. C., Malko, A., \& Lau, E. F. (2016). The electrophysiology of basic phrase building. PLOS ONE, 11(10), 122. https://doi.org/10.1371/journal.pone.0158446

Numssen, O., Zier, A.-L., Thielscher, A., Hartwigsen, G., Knösche, T. R., \& Weise, K. (2021). Efficient highresolution TMS mapping of the human motor cortex by nonlinear regression. NeuroImage, 245, 118654. https://doi.org/10.1016/j.neuroimage.2021.118654

Pallier, C., Devauchelle, A. D., \& Dehaene, S. (2011). Cortical representation of the constituent structure of sentences. Proceedings of the National Academy of Sciences of the United States of America, 108(6), 2522-2527. https : / / doi . org / 10 . 1073 / pnas . 1018711108

Poulisse, C., Wheeldon, L., Limachya, R., Mazaheri, A., \& Segaert, K. (2020). The oscillatory mechanisms associated with syntactic binding in healthy ageing. Neuropsychologia, 146, 107523. https://doi.org/10. 1016/j.neuropsychologia.2020.107523

Poulisse, C., Wheeldon, L., \& Segaert, K. (2019). Evidence Against Preserved Syntactic Comprehension in Healthy Aging. Journal of Experimental Psychology: Learning Memory and Cognition, 45(12), 2290-2308. https://doi.org/10.1037/xlm0000707

Pulvermüller, F., \& Assadollahi, R. (2007). Grammar or serial order?: discrete combinatorial brain mechanisms reflected by the syntactic mismatch negativity. Journal of cognitive neuroscience, 19(6), 97180. https://doi.org/10.1162/jocn.2007.19.6.971

Pulvermüller, F., Cook, C., \& Hauk, O. (2012). Inflection in action: semantic motor system activation to nounand verb-containing phrases is modulated by the presence of overt grammatical markers. NeuroImage, 60(2), 1367-1379. https://doi.org/10.1016/j. neuroimage.2011.12.020

Pulvermüller, F., \& Shtyrov, Y. (2003). Automatic processing of grammar in the human brain as revealed by the mismatch negativity. NeuroImage, 20(1), 159-172. https://doi.org/10.1016/S1053-8119(03)00261-1

Pulvermüller, F., \& Shtyrov, Y. (2006). Language outside the focus of attention: The mismatch negativity as a tool for studying higher cognitive processes. Progress in Neurobiology, 79(1), 49-71. https :// doi .org/10. 1016/j.pneurobio.2006.04.004

Pulvermüller, F., Shtyrov, Y., Hasting, A. S., \& Carlyon, R. P. (2008). Syntax as a reflex : Neurophysiological evidence for early automaticity of grammatical processing. Brain and Language, 104(3), 244-253. https://doi.org/https://doi-org.browser.cbs.mpg.de/ 10.1016/j.bandl.2007.05.002

Pyatigorskaya, E., Maran, M., \& Zaccarella, E. (2021). Testing the automaticity of syntax using masked visual 
priming. PsyArXiv. https://doi.org/https://doi.org/ 10.31234/osf.io/auy69

Pylkkänen, L. (2019). The neural basis of combinatory syntax and semantics. Science, 366(6461), 62-66. https://doi.org/10.1126/science.aax0050

Pylkkänen, L. (2020). Neural basis of basic composition: What we have learned from the red-boat studies and their extensions. Philosophical Transactions of the Royal Society B: Biological Sciences, 375(1791). https://doi.org/10.1098/rstb.2019.0299

Ratcliff, R. (1978). A theory of memory retrieval. Psychological Review, 85(2), 59-108. https://doi.org/10. 1037/0033-295X.85.2.59

Ratcliff, R., Gomez, P., \& Mckoon, G. (2006). A Diffusion Model Account of the Lexical Decision Task. Psychological Review, 111(1), 159-182.

Resnik, P. (1992). Left-Corner Parsing and Psychological Plausibility. Proceedings of the 14th conference on Computational Linguistics, 1, 191-197. https://doi. org/10.3115/992066.992098

Sakai, K. L., Noguchi, Y., Takeuchi, T., \& Watanabe, E. (2002). Selective priming of syntactic processing by event-related transcranial magnetic stimulation of Broca's area. Neuron, 35(6), 1177-1182. https : //doi.org/10.1016/S0896-6273(02)00873-5

Schell, M., Zaccarella, E., \& Friederici, A. D. (2017). Differential cortical contribution of syntax and semantics: An fMRI study on two-word phrasal processing. Cortex, 96, 105-120. https://doi.org/10.1016/j. cortex.2017.09.002

Schmidt, R. (1986). Was weiss der Artikel vom Hauptwort? Em Beitrag zur Verarbeitung syn- taktischer Beziehungen beim Lesen. Zeitschrift fur experimentelle and angewandte Psychologie, 33, 150163.

Schoffelen, J. M., Hultén, A., Lam, N., Marquand, A. F., Uddén, J., \& Hagoort, P. (2017). Frequency-specific directed interactions in the human brain network for language. Proceedings of the National Academy of Sciences of the United States of America, 114(30), 8083-8088. https : / / doi . org / 10 . 1073 / pnas . 1703155114

Segaert, K., Mazaheri, A., \& Hagoort, P. (2018). Binding language: structuring sentences through precisely timed oscillatory mechanisms. European Journal of Neuroscience, 48(7), 2651-2662. https://doi.org/ 10.1111/ejn.13816

Sehyr, Z. S., Caselli, N., Cohen-Goldberg, A. M., \& Emmorey, K. (2021). The ASL-LEX 2.0 Project: A Database of Lexical and Phonological Properties for 2,723 Signs in American Sign Language. Journal of deaf studies and deaf education, 26(2), 263277. https://doi.org/10.1093/deafed/enaa038
Seidenberg, M. S., Waters, G. S., Sanders, M., \& Langer, P. (1984). Pre- and postlexical loci of contextual effects on word recognition. Memory Cognition, 12(4), 315-328. https : / / doi . org / 10 . 3758 / BF03198291

Shtyrov, Y., Pulvermüller, F., Näätänen, R., \& Ilmoniemi, R. J. (2003). Grammar processing outside the focus of attention: an MEG study. Journal of cognitive neuroscience, 15(8), 1195-206. https://doi.org/ 10.1162/089892903322598148

Snijders, T. M., Petersson, K. M., \& Hagoort, P. (2010). Effective connectivity of cortical and subcortical regions during unification of sentence structure. $\mathrm{Neu}$ roImage, 52(4), 1633-1644. https:// doi .org/ 10 . 1016/j.neuroimage.2010.05.035

Steinhauer, K., \& Drury, J. E. (2012). On the early leftanterior negativity (ELAN) in syntax studies. Brain and Language, 120(2), 135-162. https://doi.org/10. 1016/j.bandl.2011.07.001

Strijkers, K., Chanoine, V., Munding, D., Dubarry, A. S., Trébuchon, A., Badier, J. M., \& Alario, F. X. (2019). Grammatical class modulates the (left) inferior frontal gyrus within 100 milliseconds when syntactic context is predictive. Scientific Reports, 9(1), 1-13. https://doi.org/10.1038/s41598-01941376-x

Stroh, A. L., Rösler, F., Dormal, G., Salden, U., Skotara, N., Hänel-Faulhaber, B., \& Röder, B. (2019). Neural correlates of semantic and syntactic processing in German Sign Language. NeuroImage, 200, 231241. https://doi.org/10.1016/j.neuroimage.2019.06. 025

Tanner, D., Morgan-Short, K., \& Luck, S. J. (2015). How inappropriate high-pass filters can produce artifactual effects and incorrect conclusions in ERP studies of language and cognition. Psychophysiology, 52(8), 997-1009. https://doi.org/10.1111/psyp.12437

Trettenbrein, P. C., Papitto, G., Friederici, A. D., \& Zaccarella, E. (2020). The functional neuroanatomy of sign language without speech. Human Brain Mapping, 1-14. https://doi.org/10.1002/hbm.25254

Trettenbrein, P. C., Pendzich, N.-K., Cramer, J.-M., Steinbach, M., \& Zaccarella, E. (2021). Psycholinguistic norms for more than 300 lexical signs in German Sign Language (DGS). Behavior Research Methods, 53(5). https://doi.org/10.3758/s13428-02001524-y

Trettenbrein, P. C., \& Zaccarella, E. (2021). Controlling Video Stimuli in Sign Language and Gesture Research: The OpenPoseR Package for Analyzing OpenPose Motion-Tracking Data in R. Frontiers in Psychology, 12, 1-7. https://doi.org/https://doi.org/ 10.3389/fpsyg.2021.628728 
Tsigka, S., Papadelis, C., Braun, C., \& Miceli, G. (2014). Distinguishable neural correlates of verbs and nouns: A MEG study on homonyms. Neuropsychologia, 54(1), 87-97. https://doi.org/10.1016/ j.neuropsychologia.2013.12.018

Tyler, L. K., Randall, B., \& Stamatakis, E. a. (2008). Cortical differentiation for nouns and verbs depends on grammatical markers. Journal of cognitive neuroscience, 20(8), 1381-1389. https://doi.org/10.1162/ jocn.2008.20095

Vaidya, A. R., Pujara, M. S., Petrides, M., Murray, E. A., \& Fellows, L. K. (2019). Lesion Studies in Contemporary Neuroscience. Trends in Cognitive Sciences, 1-19. https://doi.org/10.1016/j.tics.2019.05.009

Vigliocco, G., Vinson, D. P., Druks, J., Barber, H., \& Cappa, S. F. (2011). Nouns and verbs in the brain: A review of behavioural, electrophysiological, neuropsychological and imaging studies. Neuroscience and Biobehavioral Reviews, 35(3), 407-426. https: //doi.org/10.1016/j.neubiorev.2010.04.007

Voss, A., Rothermund, K., \& Voss, J. (2004). Interpreting the parameters of the diffusion model: An empirical validation. Memory and Cognition, 32(7), 12061220. https://doi.org/10.3758/BF03196893

Weise, K., Numssen, O., Thielscher, A., Hartwigsen, G., \& Knösche, T. R. (2020). A novel approach to localize cortical TMS effects. NeuroImage, 209. https : //doi.org/10.1016/j.neuroimage.2019.116486

Weiss, S., \& Mueller, H. M. (2012). "Too many betas do not spoil the broth": The role of beta brain oscillations in language processing. Frontiers in Psychology, 3, 1-15. https://doi.org/10.3389/fpsyg.2012.00201

West, R. F., \& Stanovich, K. E. (1982). Source of Inhibition in Experiments on the Effect of Sentence Context on Word Recognition. Journal of Experimental Psychology: Learning Memory and Cognition, 8(5), 385-399.

Widmann, A., Schröger, E., \& Maess, B. (2015). Digital filter design for electrophysiological data - a practical approach. Journal of Neuroscience Methods, 250, 3446. https://doi.org/10.1016/j.jneumeth.2014.08.002

Wu, C. Y., Zaccarella, E., \& Friederici, A. D. (2019). Universal neural basis of structure building evidenced by network modulations emerging from Broca's area: The case of Chinese. Human Brain Mapping, 40(6), 1705-1717. https://doi.org/10.1002/hbm.24482

Yu, J., \& Zhang, Y. (2008). When Chinese semantics meets failed syntax. NeuroReport, 19(7), 745-749. https: //doi.org/10.1097/WNR.0b013e3282fda21d

Zaccarella, E., \& Friederici, A. D. (2017). The neurobiological nature of syntactic hierarchies. Neuroscience and Biobehavioral Reviews, 81, 205-212. https:// doi.org/10.1016/j.neubiorev.2016.07.038
Zaccarella, E., \& Friederici, A. D. (2015a). Merge in the human brain: A sub-region based functional investigation in the left pars opercularis. Frontiers in Psychology, 6, 1-9. https://doi.org/10.3389/fpsyg. 2015.01818

Zaccarella, E., \& Friederici, A. D. (2015b). Reflections of word processing in the insular cortex: A subregional parcellation based functional assessment. Brain and Language, 142, 1-7. https://doi.org/10. 1016/j.bandl.2014.12.006

Zaccarella, E., Meyer, L., Makuuchi, M., \& Friederici, A. D. (2017). Building by Syntax: The Neural Basis of Minimal Linguistic Structures. Cerebral cortex, 27(1), 411-421. https://doi.org/10.1093/cercor/ bhv234

Zaccarella, E., Papitto, G., \& Friederici, A. D. (2021). Language and action in Broca's area: Computational differentiation and cortical segregation. Brain and Cognition, 147, 105651. https://doi.org/10.1016/j. bandc.2020.105651

Zaccarella, E., Schell, M., \& Friederici, A. D. (2017). Reviewing the functional basis of the syntactic Merge mechanism for language: A coordinate-based activation likelihood estimation meta-analysis. Neuroscience and Biobehavioral Reviews, 80, 646-656. https://doi.org/10.1016/j.neubiorev.2017.06.011

Zhang, Y., Yu, J., \& Boland, J. E. (2010). Semantics Does Not Need a Processing License From Syntax in Reading Chinese. Journal of Experimental Psychology: Learning Memory and Cognition, 36(3), 765781. https://doi.org/10.1037/a0019254

Zorzi, M., Di Bono, M. G., \& Fias, W. (2011). Distinct representations of numerical and non-numerical order in the human intraparietal sulcus revealed by multivariate pattern recognition. NeuroImage, 56(2), 674-680. https://doi.org/10.1016/j. neuroimage. 2010.06 .035 